\title{
Gerontology
}

\section{Individual Characteristics and Physical Activity in Older Adults: A Systematic Review}

\author{
Nanna Notthoff Peter Reisch Denis Gerstorf \\ Department of Psychology, Humboldt University Berlin, Berlin, Germany
}

\section{Keywords}

Physical activity · Aging · Individual characteristics · Demographics · Self-efficacy · Motivation · Locus of control $\cdot$ Health

\begin{abstract}
Background: People aged 50 years and older are regularly identified as the most sedentary group in the population. However, even within this group, there are considerable interindividual variations in physical activity (PA) levels. They have been the subject of many studies. Based on single studies, no clear picture as to which characteristics are important has emerged. Objective: The goal of our contribution was to identify which individual characteristics are consistently linked to high PA levels in older adults. Methods: We conducted a systematic review of the literature considering demographic characteristics (gender, education, marital status, employment), health (subjective, health problems), and psychological factors (motivation, self-efficacy, locus of control). A systematic search of abstracts in the database Web of Science and a thorough screening process according to a priori specified criteria yielded 63 studies for inclusion in this review. Results: Two psychological factors - motivation and self-efficacy - and the perception of one's health seem to be
\end{abstract}

\section{KARGER}

(C) 2017 S. Karger AG, Basel

E-Mail karger@karger.com

www.karger.com/ger consistently linked to higher PA levels in older adults. Selected demographic variables - gender and education - may be important for some types of PA. Conclusion: Our review suggests that differentiation of PA by domains is important for identifying and understanding which individual characteristics are associated with PA levels and how. Pinpointing what reliably distinguishes older adults who are active from those who are not is essential for designing effective interventions to promote PA in later life. @ 2017 S. Karger AG, Basel

\section{Introduction}

Active lifestyles contribute to the maintenance and improvement of health and well-being and to the prevention of diseases among older adults [1]. In particular, physical activity (PA) reduces the risk of cardiovascular disease [2] and osteoporosis [3] and improves cognitive functioning [4] and subjective well-being [5]. Properties of PA such as gait speed predict survival probabilities over up to 10 years [6], and population-level estimates suggest that if inactivity were eliminated, the average life expectancy would increase by 0.68 years worldwide [7]. Per the most widely used definition that describes PA as "any

Nanna Notthoff

Department of Psychology, Humboldt University Berlin Unter den Linden 6 DE-10099 Berlin (Germany)

E-Mail nanna.notthoff@hu-berlin.de 
bodily movement produced by skeletal muscles that results in energy expenditure" [8], benefits can be derived from PA performed for a variety of purposes (i.e., domains), including structured exercise, walking for transportation, working in a physically demanding job, and housework. Importantly, activities like walking that are particularly accessible to older adults [9] allow people to meet the World Health Organization's (WHO) recommendation to complete at least 150 minutes of moderateintensity PA per week $[10,11]$. These recommendations were made based on the growing evidence for PA's healthpromoting properties and the rising importance of physical inactivity as a risk factor for mortality [11].

The PA levels of older adults are too low worldwide, with most studies reporting that between 40 and $80 \%$ of older people do not meet $P$ A guidelines $[12,13]$. The findings on historical trends in PA engagement are inconsistent. Regular PA participation - defined as meeting the WHO PA recommendations [11] or being physically active at least five times per week - seems to have decreased in several countries $[12,14]$, probably because of reduced work-related PA [13], whereas PA levels in other countries have risen, possibly due to increases in leisure-time PA [12-14]. Consistently, people aged 55 years and older are the most sedentary group in the population [14], even when one considers walking, an activity that is popular among and easily accessible for older adults [15]. PA levels are often even lower in still older age groups, e.g., those over 65 years [15]. However, within this age group, there are considerable interindividual differences in PA levels. In an effort to pinpoint opportunities for intervention, individual characteristics related to higher PA levels have been the subject of many studies. Thus far, a set of attributes that would reliably characterize older adults who engage in higher levels of PA has not yet been identified. Findings from different studies related to a particular attribute frequently do not converge. For example, some reports suggested that married people were more physically active than unmarried people, whereas others suggested the reverse $[16,17]$. Similarly mixed results were also found for other variables such as gender [18]. This necessitates a systematic review of the literature. Previous endeavors to summarize findings in this area have focused on older adults' adherence to PA in randomized controlled trials [19], have examined determinants of initiation versus maintenance of PA rather than routine PA [20], or have not differentiated domains of PA that did not fall into the structured exercise category, even if structured exercise and other types of PA were distinguished [21].
The goal of this systematic review was to identify individual characteristics that are consistently linked to higher routine PA levels in different domains in older adults. We define individual characteristics as person-specific attributes such as demographic variables (e.g., gender, education), physical health (e.g., subjective health, number of health conditions), and psychological factors (e.g., motivation, locus of control). We chose demographic variables that may be related to knowledge about PA and its health-promoting properties (education), that may be linked to PA in specific contexts, e.g., work (employment status), and that have yielded mixed findings with regards to PA participation in previous research $[16,17]$. Our approach was aimed at being gender sensitive (inclusion of gender). The consideration of health is important because, on the one hand, it may limit people's ability to be active, but, on the other hand, it may necessitate the participation in PA aimed at prevention or rehabilitation. The chosen psychological variables are incorporated in reputable health behavior theories (motivation: e.g., Theory of Planned Behavior [22], self-determination theory [23]; self-efficacy: Social Cognitive Theory [24]; locus of control: Theory of Planned Behavior [22]). The aim of this review was to answer the question of how specific individual characteristics (gender, education, marital status, employment, physical health, motivation, self-efficacy, life satisfaction, and locus of control) of cognitively intact older adults are related to routine PA in people's daily lives.

\section{Methods}

The methodological approach was based on the PRISMA guidelines [25] developed to improve and systematize reporting standards in reviews and meta-analyses. Although this review was not comprised of randomized controlled trials, we sought to follow the PRISMA guidelines as closely as possible. A protocol for this systematic review was established and used, but not preregistered.

The a priori specified criteria for study inclusion were as follows. Samples had to include older adults aged $\geq 60$ years, the United Nations cutoff for old age [26]. Whenever samples were comprised of adults across the life span, results had to be reported separately for those at least 60 years of age. We focused on participants with normal cognitive functioning because we assumed that they could understand the health-promoting properties of PA. No restrictions were made for place of residence, i.e., participants could reside in the community, assisted living facilities, or care homes.

Due to our interest in older adult's routine PA, this systematic review does not include intervention studies. To be considered for inclusion, studies had to assess at least one of the individual characteristics of interest. For demographic variables, we included 
gender (men versus women), education (higher versus lower education), marital status (married versus not married), and employment (employed versus not employed). Physical health referred to both subjective health (better versus worse health) as well as the number of health problems (lower versus higher number) and presence of chronic health conditions (healthy versus chronic health condition). The psychological factors examined were motivation (higher versus lower), self-efficacy (higher versus lower), life satisfaction (higher versus lower), and locus of control (internal versus external).

The outcome was PA as defined earlier [8], comprehensively encompassing different domains: structured exercise and sports; walking; PA in house or garden; work-related PA; transportationrelated PA; leisure-time PA; light PA; moderate PA; vigorous PA; a composite consisting of moderate and vigorous $\mathrm{PA}$; meeting the WHO or similar PA guidelines (150 min of moderate aerobic activity per week or 75 min of vigorous aerobic activity per week and strength training twice a week [11]); and total PA that was not specifically classified into any of the previously listed categories or referred to activity from more than one category. PA level was treated on a continuum, whenever possible. When studies dichotomized PA (e.g., meeting PA guidelines: yes versus no), the same classification was adopted. This review focuses on associations between individual characteristics and the level of PA, not the change in PA over time; longitudinal components of the included studies were thus not considered. PA had to be measured over a period of at least 7 days, either with an accelerometer or pedometer or with a questionnaire that referred to a period of $\geq 7$ days. Initial estimates applying a classical measurement error model and requiring the intraclass correlation coefficient to be $>0.80$ have suggested that the 7-day measurement period sufficiently minimizes intraindividual variations in PA when PA is monitored objectively with pedometers or accelerometers [27, 28]. When questionnaires are used to measure $\mathrm{PA}$, recall error can be reduced when participants are asked to report on recent engagement in PA and when recall is clearly structured [29]. The 7-day period is advantageous because it allows assessment of PA on both weekdays and weekend days, which ensures that activities that go beyond daily self-care behaviors are captured $[28,29]$.

To identify studies, we systematically searched abstracts in the scientific database Web of Science using the following search terms: physical activity; exercise; walking; sports; older adults; seniors; elderly; old age; gender; education; marital status; occupation; health; motivation; self-efficacy; life satisfaction; satisfaction with life; locus of control; control beliefs. The search was restricted by excluding studies containing the following search terms: intervention; cognitive impairment; cognitively impaired; dementia; Alzheimer. The detailed search strategy and combination of search terms is presented in the online supplementary material (see www.karger.com/doi/10.1159/000475558). Only studies published in peer-reviewed journals in English or German between January 1995 and September 2016 were considered. The literature search was conducted between April 2016 and September 2016 by the first and second author.

Eligibility was assessed by the first and second author; interrater reliability was $\kappa=0.98$; in cases of disagreement, the first author made the final call. Data were extracted from the selected full-text articles by giving a score of "+" when the association between the examined individual characteristic and PA was positive, a score of "0" when there was no association, and a score of “-”

Individual Characteristics and Physical Activity when the association was negative. For the majority of the variables, associations between individual characteristics and PA levels were understood to lie on a continuum. Most individual characteristics were defined as continuous variables in the original studies. For example, a significant positive association between motivation and PA means that higher levels of motivation were associated with higher levels of PA; it does not refer to a comparison of PA engagement for "motivated" and "unmotivated" people. For the categorical variables, we adopted the categories from the original studies. Scores were assigned as follows: For gender, a score of "+" indicated more activity in men than in women, " 0 " equal activity in both genders, and "-" less activity in men than in women. For marital status, "+” referred to more activity in married than in unmarried (e.g., never married, widowed, divorced, separated) individuals, " 0 " to equal amounts of activity in married and unmarried individuals, and "-" to less activity in married than unmarried individuals. With regards to employment, "+" was given when employed individuals were more active than not employed individuals (e.g., retired, unemployed), "0" when employed and not employed individuals were equally active, and "-" when employed individuals were less active than not employed individuals. For example, a study showing that higher levels of self-efficacy were related to more leisure-time PA would have received a score of "+"; a study showing that participants with osteoarthritis performed as much PA in the house and garden as healthy participants would have received a score of "0"; and a study showing that men walked less than women would have received a score of "-." Scores for each predictor-outcome pair were summarized by summing the “+," "0," and “-” scores, respectively. With this approach, we calculated how frequently a given effect was found and thus went beyond a narrative summary of the evidence, making use of a means to quantitatively summarize study findings. We acknowledge that this strategy did not allow us to grasp the size of a given effect.

The risk of bias for each study was rated by the first and second author. Interrater agreement was $\kappa=0.62$. According to Landis and Koch [30], this corresponds to substantial agreement; conservative approaches consider it moderate agreement [31]. In cases of disagreement, the final call was made by the first author. Since the studies comprising this review were not randomized controlled trials, the rating items recommended in the PRISMA guidelines could not be used. Instead, we developed rating items based on the American Psychological Association's recommendations for reporting research findings [32]. The system for rating study quality together with the study information provided in $\mathrm{Ta}$ ble 1 [33-120] meet the recommendations regarding the assessment of study quality made in the MOOSE guidelines [121] and the STROBE statement [122]. We rated each study on its research methodology (items: "Was the study sample adequately described?"; "Was the sample representative of the population being studied?"; "Was a rationale for the sample size given?"; "Were eligibility criteria for participant selection used?"; "Were withdrawals reported and explained?"; "Were measurement instruments clearly described?"; "Was a standardized study protocol used?"), statistical analyses ("Were appropriate statistical methods used?"; "Were confounding variables controlled?"), and discussion of the results ("Were limitations acknowledged?"). Each item was scored on a three-point scale with $1=$ yes, $0=$ unclear, and $-1=$ no. These ratings provided some insight into the quality of the included studies. 
Table 1. Study information

\begin{tabular}{|c|c|c|c|c|c|}
\hline $\begin{array}{l}\text { Reference } \\
\text { (first author) }\end{array}$ & Participants & $\begin{array}{l}\text { Individual charac- } \\
\text { teristics assessed }\end{array}$ & Type of PA & Measurement of PA & Results \\
\hline $\begin{array}{l}\text { Arcury } \\
{[33]}\end{array}$ & $\begin{array}{l}698 \text { older adults } \geq 65 \text { years } \\
(\mathrm{M}=74.1, \mathrm{SD}=5.4)(\text { age } \\
\text { range not given })\end{array}$ & $\begin{array}{l}\text { gender, education, } \\
\text { health (health } \\
\text { problems, } \\
\text { subjective) }\end{array}$ & total PA & $\begin{array}{l}\text { self-reported PA in the past year (exercised at least once } \\
\text { per week on average in the past year); self-reported } \\
\text { number of days physically active for at least } 30 \mathrm{~min} \text { at a } \\
\text { time }(0-7) \text { (no reference provided) }\end{array}$ & $\begin{array}{l}\text { gender: } 0 \text {, education: } 0 \text {, health } \\
\text { (health problems): }+ \text {, health } \\
\text { (subjective): } 0\end{array}$ \\
\hline $\begin{array}{l}\text { Berger } \\
{[34]}\end{array}$ & $\begin{array}{l}699 \text { older adults } \geq 60 \text { years } \\
\text { (age range or other } \\
\text { statistics not given) }\end{array}$ & $\begin{array}{l}\text { gender, education, } \\
\text { employment } \\
\text { status, health } \\
\text { (subjective) }\end{array}$ & total PA & $\begin{array}{l}\text { structured interview: location of PA (work, home, } \\
\text { leisure); time spent on physically demanding work, } \\
\text { number of stairs climbed per day, episodes of vigorous } \\
\text { PA of at least } 20 \text { min, number of working days, } \\
\text { housework, gardening, sports, games, other exercises; } \\
\text { self-reports of frequency, duration, intensity (no } \\
\text { reference provided) }\end{array}$ & $\begin{array}{l}\text { gender: }+ \text {; education: } 0 \text {; employment } \\
\text { status: }+ \text {; health (subjective): }+\end{array}$ \\
\hline $\begin{array}{l}\text { Biernat } \\
{[35]}\end{array}$ & $\begin{array}{l}262 \text { older adults } 60-69 \\
\text { years (age mean or other } \\
\text { statistics not given) }\end{array}$ & gender, education & $\begin{array}{l}\text { meeting PA } \\
\text { guidelines }\end{array}$ & $\begin{array}{l}\text { questionnaire developed and pilot-tested for this study: } \\
\text { recreational and touristic activity in the last year as well } \\
\text { as short version of the IPAQ; Cerin et al. [36] }\end{array}$ & gender: 0 ; education: + \\
\hline $\begin{array}{l}\text { Bird } \\
{[37]}\end{array}$ & $\begin{array}{l}362 \text { older adults } \geq 60 \text { years } \\
(M=72, S D=7)\end{array}$ & gender & walking & questionnaire: IPAQ; Cerin et al. [36] & gender: 0 \\
\hline $\begin{array}{l}\text { Black } \\
{[38]}\end{array}$ & $\begin{array}{l}1,976 \text { older adults } 60-64 \\
\text { years }(\mathrm{M}=62.8, \mathrm{SD}=1.2)\end{array}$ & gender & walking & $\begin{array}{l}\text { questionnaire: modified version of the EPAQ2; } \\
\text { Wareham et al. [39] }\end{array}$ & gender: - \\
\hline $\begin{array}{l}\text { Casado-Pérez } \\
{[40]}\end{array}$ & $\begin{array}{l}10,373 \text { older adults } \\
\geq 65 \text { years (means for the } \\
2 \text { years from which data } \\
\text { were drawn: } 2006: M= \\
74.46 ; 2011: M=75 \text { ) }\end{array}$ & $\begin{array}{l}\text { education, health } \\
\text { (chronic illness, } \\
\text { subjective) }\end{array}$ & LTPA & $\begin{array}{l}\text { questionnaire: "Do you practice any PA during your } \\
\text { leisure time?" (response options: "none" or "once a } \\
\text { month or more") }\end{array}$ & $\begin{array}{l}\text { education: }+ \text {; health (chronic illness): } \\
+ \text {; health (subjective): + }\end{array}$ \\
\hline $\begin{array}{l}\text { Chen } \\
{[41]}\end{array}$ & $\begin{array}{l}384 \text { older adults } 65-101 \\
\text { years }(\mathrm{M}=79.2, \mathrm{SD}=8.5)\end{array}$ & $\begin{array}{l}\text { health (chronic } \\
\text { illness) }\end{array}$ & total PA & questionnaire: not specified & health (health problems): + \\
\hline $\begin{array}{l}\text { Conn } \\
{[42]}\end{array}$ & $\begin{array}{l}147 \text { older adults } 65-100 \\
\text { years }(\mathrm{M}=78.53, \mathrm{SD}= \\
8.65)\end{array}$ & $\begin{array}{l}\text { health (subjective), } \\
\text { self-efficacy }\end{array}$ & total PA & $\begin{array}{l}\text { questionnaire: Baecke Physical Activity Scale; Baecke et } \\
\text { al. [43] }\end{array}$ & health (subjective): 0; self-efficacy: + \\
\hline $\begin{array}{l}\text { Danon-Hersch } \\
{[44]}\end{array}$ & $\begin{array}{l}1,422 \text { older adults } 65-70 \\
\text { years }\end{array}$ & gender, education & sports & $\begin{array}{l}\text { questionnaire: two adapted questions from the } \\
\text { Monitoring of Trends and Determinants in } \\
\text { Cardiovascular Disease Physical Activity Questionnaire; } \\
\text { Sequeira et al. [45]; Wietlisbach et al. [46] }\end{array}$ & gender: 0 ; education: + \\
\hline $\begin{array}{l}\text { de Souto } \\
\text { Barreto } \\
{[47]}\end{array}$ & $\begin{array}{l}393 \text { older adults } \geq 60 \text { years } \\
(\mathrm{M}=70.1, \mathrm{SD}=8) \text { (age } \\
\text { range not given })\end{array}$ & $\begin{array}{l}\text { gender, health } \\
\text { (subjective) }\end{array}$ & $\begin{array}{l}\text { meeting PA } \\
\text { guidelines }\end{array}$ & questionnaire: QAPPA; Barreto et al. [48] & gender: 0 ; health (subjective): + \\
\hline $\begin{array}{l}\text { Egerton } \\
{[49]}\end{array}$ & $\begin{array}{l}1,567 \text { older adults }(\mathrm{M}= \\
73.4, \mathrm{SD}=1.9) \text { (age range } \\
\text { not given) }\end{array}$ & $\begin{array}{l}\text { gender, health } \\
\text { (health problems) }\end{array}$ & total PA & accelerometer: ActiGraph GT3X worn on the right hip & $\begin{array}{l}\text { gender: } 0 \text {; health (health problems): } \\
0\end{array}$ \\
\hline $\begin{array}{l}\text { Ferreira } \\
{[50]}\end{array}$ & $\begin{array}{l}1,667 \text { older adults } \geq 65 \\
\text { years }(\mathrm{M}=74.9, \mathrm{SD}=6.7) \\
\text { (age range not given) }\end{array}$ & gender, education & total PA & $\begin{array}{l}\text { questionnaire: frequency (times per week), average } \\
\text { duration (minutes per session) of PA }\end{array}$ & gender: -; education: 0 \\
\hline $\begin{array}{l}\text { Gao } \\
{[51]}\end{array}$ & $\begin{array}{l}2,839 \text { older adults } \geq 60 \\
\text { years (age range or other } \\
\text { statistics not given) }\end{array}$ & marital status & $\begin{array}{l}\text { meeting PA } \\
\text { guidelines }\end{array}$ & $\begin{array}{l}\text { questionnaire: Chinese long form of the IPAQ; } \\
\text { Macfarlane et al. [52] }\end{array}$ & marital status: + \\
\hline $\begin{array}{l}\text { Giuli } \\
{[53]}\end{array}$ & $\begin{array}{l}306 \text { older adults } \geq 65 \text { years } \\
(\mathrm{M}=76.9, \mathrm{SD}=8.5)\end{array}$ & $\begin{array}{l}\text { gender, education, } \\
\text { marital status }\end{array}$ & total PA & $\begin{array}{l}\text { questionnaire: Lifestyle Questionnaire; Marcellini et al. } \\
\text { [54] }\end{array}$ & $\begin{array}{l}\text { gender: } 0 \text {; education: +: education: } 0 \text {; } \\
\text { marital status: +; marital status: } 0\end{array}$ \\
\hline $\begin{array}{l}\text { Grant-Savela } \\
{[55]}\end{array}$ & $\begin{array}{l}197 \text { older adults } 60-96 \\
\text { years }(\mathrm{M}=71.5, \mathrm{SD}=8.3)\end{array}$ & $\begin{array}{l}\text { health (health } \\
\text { problems, } \\
\text { subjective), self- } \\
\text { efficacy, } \\
\text { motivation }\end{array}$ & walking & $\begin{array}{l}\text { questionnaire: PASE; New England Research Institutes } \\
\text { [56] }\end{array}$ & $\begin{array}{l}\text { health (health problems): +; health } \\
\text { (subjective): +; self-efficacy: +; } \\
\text { motivation: + }\end{array}$ \\
\hline $\begin{array}{l}\text { Grimby } \\
{[57]}\end{array}$ & $\begin{array}{l}701 \text { older adults } \geq 65 \text { years } \\
\text { divided into three groups } \\
\text { (group } 1: M=76 \text {; group } 3 \text { : } \\
M=76 \text { ) (further age } \\
\text { statistics not given) }\end{array}$ & $\begin{array}{l}\text { marital status, } \\
\text { health (health } \\
\text { problems, } \\
\text { subjective) }\end{array}$ & walking & $\begin{array}{l}\text { questionnaire (group 2); structured interview with the } \\
\text { same questions (groups } 1 \text { and } 3 \text { ): average walking time } \\
\text { per day in min }(0-15,15-30,30-60,60-120,120+) \text {; } \\
\text { average number of days per week with that walking time } \\
(1-2,3-4,5-6 \text {; a few times a month }=0.5 \text {; nearly never } \\
=0) \text { (no reference provided) }\end{array}$ & $\begin{array}{l}\text { marital status: } 0 \text {; health (health } \\
\text { problems): } 0 \text {; health(subjective): +; } \\
\text { health (subjective): } 0\end{array}$ \\
\hline $\begin{array}{l}\text { Herbolsheimer } \\
{[58]}\end{array}$ & $\begin{array}{l}2,942 \text { older adults } 65-85 \\
\text { years (age mean and other } \\
\text { statistics not given) }\end{array}$ & $\begin{array}{l}\text { health (health } \\
\text { problems) }\end{array}$ & $\begin{array}{l}\text { walking, PA } \\
\text { in house/ } \\
\text { garden, } \\
\text { sports, total } \\
\text { PA }\end{array}$ & questionnaire: LAPAQ (no reference provided) & $\begin{array}{l}\text { health (health problems) on walking: } \\
\text { +; health (health problems) on PA in } \\
\text { house/garden: } 0 \text {; health (health } \\
\text { problems) on sports: } 0 \text {; health } \\
\text { (health problems) on total PA: } 0\end{array}$ \\
\hline
\end{tabular}


Table 1 (continued)

\begin{tabular}{|c|c|c|c|c|c|}
\hline $\begin{array}{l}\text { Reference } \\
\text { (first author) }\end{array}$ & Participants & $\begin{array}{l}\text { Individual charac- } \\
\text { teristics assessed }\end{array}$ & Type of PA & Measurement of PA & Results \\
\hline $\begin{array}{l}\text { Hirakawa } \\
{[59]}\end{array}$ & $\begin{array}{l}324 \text { older adults, of these } \\
172 \text { older adults } \geq 65 \text { years } \\
\text { (age mean or other } \\
\text { statistics not given) }\end{array}$ & health (subjective) & total PA & $\begin{array}{l}\text { questionnaire: exercise habits (minimum of two } 30-\mathrm{min} \\
\text { sessions of exercise per week) (no reference provided) }\end{array}$ & health (subjective): 0 \\
\hline $\begin{array}{l}\text { Hirvensalo } \\
{[60]}\end{array}$ & $\begin{array}{l}1,224 \text { older adults } 65-84 \\
\text { years (age mean or other } \\
\text { statistics not given) }\end{array}$ & gender & total PA & $\begin{array}{l}\text { questionnaire: participation in calisthenic exercises } \\
\text { at home, swimming, cycling, cross-country skiing, } \\
\text { dancing, supervised physical exercise classes, gym } \\
\text { training, ball games, other (6-point scale from daily to } \\
\text { nonparticipation); walking for fitness (almost daily, } 1-3 \\
\text { times a week, rarely or never) (no reference provided) }\end{array}$ & $\begin{array}{l}\text { gender: + (young-old); gender: } 0 \\
\text { (old-old) }\end{array}$ \\
\hline $\begin{array}{l}\text { Hughes } \\
{[61]}\end{array}$ & $\begin{array}{l}5,589 \text { older adults } \geq 60 \\
\text { years (age mean or other } \\
\text { statistics not given) }\end{array}$ & $\begin{array}{l}\text { gender, education, } \\
\text { marital status, } \\
\text { health (subjective) }\end{array}$ & LTPA & $\begin{array}{l}\text { questionnaire: LTPA section of the PAQ from the } \\
\text { NHANES; Centers for Disease Control and Prevention } \\
\text { [62] }\end{array}$ & $\begin{array}{l}\text { gender: +; education: +; marital } \\
\text { status: } 0 \text {; health (subjective): }+\end{array}$ \\
\hline $\begin{array}{l}\text { Huisingh- } \\
\text { Scheetz } \\
{[63]}\end{array}$ & $\begin{array}{l}3,196 \text { older adults } 62-91 \\
\text { years; accelerometer } \\
\text { subsample: } 738 \text { older } \\
\text { adults (age mean or other } \\
\text { statistics not given) }\end{array}$ & gender & total PA & $\begin{array}{l}\text { questionnaire: four questions about, e.g., participation } \\
\text { in vigorous PA of } \geq 30 \text { min ( } 5+\text { times per week, } 3-4 \\
\text { times per week, } 1-2 \text { times per week, } 1-3 \text { times per } \\
\text { month, less than once a month, never); other questions } \\
\text { not specified (no reference provided); accelerometer: } \\
\text { Actiwatch Spectrum worn on the wrist }\end{array}$ & $\begin{array}{l}\text { questionnaire: gender: +; } \\
\text { accelerometer: gender: - }\end{array}$ \\
\hline $\begin{array}{l}\text { Ismail } \\
{[64]}\end{array}$ & $\begin{array}{l}408 \text { older adults } \geq 60 \text { years } \\
(\mathrm{M}=66.4, \mathrm{SD}=5.6)\end{array}$ & health (subjective) & total PA & $\begin{array}{l}\text { questionnaire: PASE-M; New England Research } \\
\text { Institutes [56]; Washburn et al. [65] }\end{array}$ & health (subjective): + \\
\hline $\begin{array}{l}\text { Jerome } \\
{[66]}\end{array}$ & $\begin{array}{l}710 \text { older adults } 70-79 \\
\text { years (age mean or other } \\
\text { statistics not given) }\end{array}$ & $\begin{array}{l}\text { health (health } \\
\text { problems) }\end{array}$ & $\begin{array}{l}\text { meeting PA } \\
\text { guidelines }\end{array}$ & $\begin{array}{l}\text { questionnaire: modified version of the Minnesota } \\
\text { Leisure Time Physical Activity Questionnaire; Folsom et } \\
\text { al. [67]; Taylor et al. [68] }\end{array}$ & health (health problems): + \\
\hline $\begin{array}{l}\text { Johansson } \\
\text { [69] }\end{array}$ & $\begin{array}{l}1,390 \text { older adults aged } \\
70 \text { years }\end{array}$ & gender & total PA & $\begin{array}{l}\text { accelerometer: ActiGraph GT3X+ worn on the } \\
\text { nondominant hip }\end{array}$ & gender: 0 \\
\hline $\begin{array}{l}\text { Kahana } \\
{[70]}\end{array}$ & $\begin{array}{l}453 \text { older adults } 72-98 \\
\text { years }(\mathrm{M}=79.13, \mathrm{SD}= \\
4.13)\end{array}$ & gender & sports & $\begin{array}{l}\text { questionnaire: hours per week spent walking, } \\
\text { swimming, golfing, running/jogging, aerobics, } \\
\text { stretching or calisthenics, weight lifting, dancing, biking, } \\
\text { other exercises (no reference provided) }\end{array}$ & gender: + \\
\hline $\begin{array}{l}\text { Kaur } \\
{[71]}\end{array}$ & $\begin{array}{l}4,831 \text { older adults } \geq 60 \\
\text { years (age range or other } \\
\text { statistics not given) }\end{array}$ & $\begin{array}{l}\text { gender, education, } \\
\text { marital status }\end{array}$ & total PA & $\begin{array}{l}\text { questionnaire: core component of the PA module of the } \\
\text { WHO STEPS Instrument; WHO [72] }\end{array}$ & $\begin{array}{l}\text { gender: +; education: } 0 \text {; marital } \\
\text { status: } 0\end{array}$ \\
\hline $\begin{array}{l}\text { Kendig } \\
{[73]}\end{array}$ & $\begin{array}{l}1,422 \text { older adults } \geq 65 \\
\text { years (age range or other } \\
\text { statistics not given) }\end{array}$ & gender, education & total PA & $\begin{array}{l}\text { questionnaire: participants were asked whether they had } \\
\text { engaged in "energetic" PA during the last } 2 \text { weeks; they } \\
\text { could list up to three activities from the categories } \\
\text { sports, walking, home maintenance, housework, } \\
\text { gardening; the method was based on the Australian Risk } \\
\text { Factor Prevalence Study; Risk Factor Prevalence } \\
\text { Management Committee [74] }\end{array}$ & gender: 0 ; education: 0 \\
\hline $\begin{array}{l}\text { Kerr } \\
{[75]}\end{array}$ & $\begin{array}{l}896 \text { older adults } \geq 66 \text { years } \\
\text { (age mean or other } \\
\text { statistics not given) }\end{array}$ & health (subjective) & general PA & $\begin{array}{l}\text { questionnaire: frequency of PA in different locations } \\
\text { (indoors at home or apartment building, other indoor } \\
\text { settings like recreation facilities, outdoors in a green or } \\
\text { open space, outdoors in local streets or neighborhood, } \\
\text { outdoors outside of local neighborhood), response } \\
\text { options: more than once a week, once a week, less than } \\
\text { once a week; accelerometer: ActiGraph (not specified } \\
\text { where worn) }\end{array}$ & health (subjective): + \\
\hline $\begin{array}{l}\text { Lawlor } \\
{[76]}\end{array}$ & $\begin{array}{l}2,341 \text { older adults } 60-79 \\
\text { years (age mean or other } \\
\text { statistics not given) }\end{array}$ & $\begin{array}{l}\text { marital status, } \\
\text { health (health } \\
\text { problems, } \\
\text { subjective) }\end{array}$ & $\begin{array}{l}\text { walking, PA } \\
\text { in house/ } \\
\text { garden, total } \\
\text { PA }\end{array}$ & $\begin{array}{l}\text { questionnaire: adapted from the British Heart Study and } \\
\text { available in the supplementary material: usual duration } \\
\text { of activity in hours per week for walking, cycling, } \\
\text { physical exercise, light and heavy housework and } \\
\text { gardening, do it yourself }\end{array}$ & $\begin{array}{l}\text { marital status on walking: 0; marital } \\
\text { status on PA in house/garden: } 0 \text {; } \\
\text { marital status on total PA: } 0 \text {; health } \\
\text { (health problems) on walking: } \\
3 \text { times + and } 2 \text { times } 0 \text {; health } \\
\text { (health problems) on PA in house/ } \\
\text { garden: } 3 \text { times + and } 2 \text { times } 0 \text {; } \\
\text { health (health problems) on total PA: } \\
+ \text { and } 4 \text { times 0; health (subjective) } \\
\text { on walking: +; health (subjective) on } \\
\text { PA in house/garden: +; health } \\
\text { (subjective) on total PA: + }\end{array}$ \\
\hline $\begin{array}{l}\text { Lee } \\
{[77]}\end{array}$ & $\begin{array}{l}276 \text { older adults } 60-75 \\
\text { years }(M=69, S D=4.11)\end{array}$ & gender & $\begin{array}{l}\text { walking, } \\
\text { LTPA }\end{array}$ & questionnaire: HPAQ; Voorrips et al. [78] & $\begin{array}{l}\text { gender on walking frequency: } 0 \text {; } \\
\text { gender on walking duration: +; } \\
\text { gender on LTPA frequency: } 0 \text {; } \\
\text { gender on LTPA duration: + }\end{array}$ \\
\hline
\end{tabular}


Table 1 (continued)

\begin{tabular}{|c|c|c|c|c|c|}
\hline $\begin{array}{l}\text { Reference } \\
\text { (first author) }\end{array}$ & Participants & $\begin{array}{l}\text { Individual charac- } \\
\text { teristics assessed }\end{array}$ & Type of PA & Measurement of PA & Results \\
\hline $\begin{array}{l}\text { Lee and } \\
\text { Laffrey } \\
{[79]}\end{array}$ & $\begin{array}{l}267 \text { older adults } 60-75 \\
\text { years }(M=69, S D=4.12)\end{array}$ & $\begin{array}{l}\text { gender, health } \\
\text { (subjective), self- } \\
\text { efficacy }\end{array}$ & total PA & questionnaire: HPAQ; Voorrips et al. [78] & $\begin{array}{l}\text { gender: +; health (subjective): } 0 \text {; } \\
\text { self-efficacy: }+\end{array}$ \\
\hline $\begin{array}{l}\text { Leveille } \\
{[80]}\end{array}$ & $\begin{array}{l}328 \text { older adults } 75-85 \\
\text { years (age mean or other } \\
\text { statistics not given) }\end{array}$ & $\begin{array}{l}\text { self-efficacy, locus } \\
\text { of control }\end{array}$ & walking & questionnaire: PASE; Washburn et al. [65] & self-efficacy: +; locus of control: 0 \\
\hline $\begin{array}{l}\text { Lian } \\
{[81]}\end{array}$ & $\begin{array}{l}2,494 \text { older adults } \geq 60 \\
\text { years (age mean or other } \\
\text { statistics not given) }\end{array}$ & gender, education & LTPA & $\begin{array}{l}\text { questionnaire: weekly frequency of vigorous or } \\
\text { moderate activity lasting at least } 20 \mathrm{~min} \text { (no reference } \\
\text { provided) }\end{array}$ & $\begin{array}{l}\text { gender: +; education: }+ \text { for men and } \\
0 \text { for women }\end{array}$ \\
\hline $\begin{array}{l}\text { Lim and } \\
\text { Taylor } \\
{[82]}\end{array}$ & $\begin{array}{l}8,881 \text { older adults } \geq 65 \\
\text { years }(M=73.8) \text { (age } \\
\text { range or other statistics } \\
\text { not given) }\end{array}$ & $\begin{array}{l}\text { gender, } \\
\text { employment, } \\
\text { health (health } \\
\text { problems) }\end{array}$ & total PA & $\begin{array}{l}\text { questionnaire: number of days participants spent } \\
\text { walking, doing moderate activities like dancing, golf, } \\
\text { lawn bowls, and vigorous gardening or yard work (no } \\
\text { reference provided) }\end{array}$ & $\begin{array}{l}\text { gender: +; employment: } 0 \text {; health } \\
\text { (health problems): + }\end{array}$ \\
\hline $\begin{array}{l}\text { Loland } \\
{[83]}\end{array}$ & $\begin{array}{l}3,770 \text { older adults } 65-97 \\
\text { years }(\mathrm{M}=75.05, \mathrm{SD}=5.8)\end{array}$ & $\begin{array}{l}\text { education, marital } \\
\text { status }\end{array}$ & sports & questionnaire: PASE; Washburn et al. [65] & education: +; marital status: 0 \\
\hline $\begin{array}{l}\text { Mäkilä } \\
{[84]}\end{array}$ & $\begin{array}{l}635 \text { older adults } \geq 65 \text { years } \\
\text { (year } 1988: M=69 ; \text { year } \\
1996: M=76 \text {; year } 2004: \\
M=84 \text { ) (age range or } \\
\text { other statistics not given) }\end{array}$ & gender & total PA & $\begin{array}{l}\text { questionnaire: frequency and intensity of different types } \\
\text { of PA (no reference provided) }\end{array}$ & gender: + \\
\hline $\begin{array}{l}\text { Menec and } \\
\text { Chipperfield } \\
{[85]}\end{array}$ & $\begin{array}{l}1,258 \text { older adults } 60-95 \\
\text { years }(M=69.6)(S D \text { not } \\
\text { given })\end{array}$ & $\begin{array}{l}\text { gender, education, } \\
\text { health (health } \\
\text { problems), locus of } \\
\text { control }\end{array}$ & sports & $\begin{array}{l}\text { questionnaire: engagement in different activities over } \\
\text { the last week; time spent exercising during } 1 \text { week (no } \\
\text { exercise, } 1-60 \mathrm{~min}, 61-120 \mathrm{~min}, \ldots,>9 \mathrm{~h} \text { ) (reference } \\
\text { not provided) }\end{array}$ & $\begin{array}{l}\text { gender: +; education: } 0 \text {; health } \\
\text { (health problems): }+ \text {; locus of control } \\
\text { (general): }+ \text {; locus of control (health): } \\
0\end{array}$ \\
\hline $\begin{array}{l}\text { Merom } \\
{[86]}\end{array}$ & $\begin{array}{l}22,050 \text { older adults } \geq 65 \\
\text { years (age range or other } \\
\text { statistics not given) }\end{array}$ & $\begin{array}{l}\text { gender, education, } \\
\text { health (subjective) }\end{array}$ & sports & questionnaire: ERASS; Merom et al. [87] & $\begin{array}{l}\text { gender: +; education: }+ \text {; health } \\
\text { (subjective): }+\end{array}$ \\
\hline $\begin{array}{l}\text { Mier } \\
{[88]}\end{array}$ & $\begin{array}{l}238 \text { older adults } \geq 60 \text { years } \\
\text { (age range or other } \\
\text { statistics not given) }\end{array}$ & $\begin{array}{l}\text { gender, education, } \\
\text { health (health } \\
\text { problems) }\end{array}$ & $\begin{array}{l}\text { meeting PA } \\
\text { guidelines }\end{array}$ & $\begin{array}{l}\text { questionnaire: number of days with PA of at least } \\
30 \mathrm{~min} \text { in the last week (no reference provided) }\end{array}$ & $\begin{array}{l}\text { gender: +; education: }+ \text {; health } \\
\text { (health problems): } 0\end{array}$ \\
\hline $\begin{array}{l}\text { Moschny } \\
{[89]}\end{array}$ & $\begin{array}{l}6,880 \text { older adults } 72-93 \\
\text { years }(\mathrm{MD}=77 ; \mathrm{M} \text { and } \mathrm{SD} \\
\text { not given })\end{array}$ & gender, education & $\begin{array}{l}\text { sports, PA } \\
\text { in house/ } \\
\text { garden }\end{array}$ & questionnaire: PRISCUS-PAQ; Trampisch et al. [90] & $\begin{array}{l}\text { gender on sports: +; gender on PA } \\
\text { in house/garden: -; education on } \\
\text { sports: 0; education on PA in house/ } \\
\text { garden: + }\end{array}$ \\
\hline $\begin{array}{l}\text { Murphy } \\
{[91]}\end{array}$ & $\begin{array}{l}4,663 \text { participants, of these } \\
561 \text { older adults } \geq 61 \text { years } \\
\text { (no age range or other } \\
\text { statistics given) }\end{array}$ & gender & $\begin{array}{l}\text { PA in house/ } \\
\text { garden }\end{array}$ & $\begin{array}{l}\text { questionnaire: modified version of the Active People } \\
\text { Survey (Sport England) (no specific reference provided) }\end{array}$ & gender on PA in house/garden: - \\
\hline $\begin{array}{l}\text { Mynarski } \\
{[92]}\end{array}$ & $\begin{array}{l}456 \text { adults, of these one } \\
\text { group }>60 \text { to } 65 \text { years } \\
\text { (number not specified; age } \\
\text { mean or other statistics not } \\
\text { given) }\end{array}$ & gender & $\begin{array}{l}\text { meeting PA } \\
\text { guidelines }\end{array}$ & $\begin{array}{l}\text { questionnaire: short version of the IPAQ; Cerin et al. } \\
\text { [36] }\end{array}$ & gender: 0 \\
\hline $\begin{array}{l}\text { Palacios-Ceña } \\
\text { [93] }\end{array}$ & $\begin{array}{l}29,263 \text { older adults } \geq 65 \\
\text { years (data from different } \\
\text { years were collapsed, age } \\
\text { mean between } 72 \text { and } 75, \\
\text { range and SD not } \\
\text { provided) }\end{array}$ & $\begin{array}{l}\text { education, marital } \\
\text { status, health } \\
\text { (health problems, } \\
\text { subjective) }\end{array}$ & LTPA & $\begin{array}{l}\text { questionnaire: any LTPA (none, once a month, or more) } \\
\text { (no reference provided) }\end{array}$ & $\begin{array}{l}\text { education: } 0 \text {; marital status: } 0 \text {; health } \\
\text { (health problems): } 2 \text { times }+, 1 \text { time } \\
0 \text {; health (subjective): } 0\end{array}$ \\
\hline $\begin{array}{l}\text { Persson } \\
{[94]}\end{array}$ & $\begin{array}{l}255 \text { older adults } 60-99 \\
\text { years }(\mathrm{M}=75, \mathrm{SD}=8.79)\end{array}$ & $\begin{array}{l}\text { gender, education, } \\
\text { health (subjective) }\end{array}$ & $\begin{array}{l}\text { meeting PA } \\
\text { guidelines }\end{array}$ & $\begin{array}{l}\text { questionnaire: participation in moderate and vigorous } \\
\text { activity during a week, seemed to be based on PASE; } \\
\text { Washburn et al. [65] }\end{array}$ & $\begin{array}{l}\text { gender: } 0 \text {; education: } 0 \text {; health } \\
\text { (subjective): + and } 0\end{array}$ \\
\hline $\begin{array}{l}\text { Reigal } \\
{[95]}\end{array}$ & $\begin{array}{l}289 \text { older adults } 65-85 \\
\text { years }(\mathrm{M}=74.15, \mathrm{SD}= \\
5.71)\end{array}$ & self-efficacy & total PA & $\begin{array}{l}\text { questionnaire: weekly participation in PA (no reference } \\
\text { provided) }\end{array}$ & self-efficacy: 0 \\
\hline $\begin{array}{l}\text { Resnick } \\
{[96]}\end{array}$ & $\begin{array}{l}59 \text { older adults } \geq 65 \text { years } \\
(\mathrm{M}=88, \mathrm{SD}=6.9) \text { (age } \\
\text { range not given) }\end{array}$ & $\begin{array}{l}\text { gender, marital } \\
\text { status, health } \\
\text { (subjective), self- } \\
\text { efficacy }\end{array}$ & sports & $\begin{array}{l}\text { questionnaire: participation in exercise for at least } \\
20 \text { min at a time at least } 3 \text { times per week (self-report, } \\
\text { records from nursing staff) }\end{array}$ & $\begin{array}{l}\text { gender: } 0 \text {; marital status: } 0 \text {; health } \\
\text { (subjective): } 0 \text {; self-efficacy: }+\end{array}$ \\
\hline $\begin{array}{l}\text { Resnick } \\
{[97]}\end{array}$ & $\begin{array}{l}175 \text { older adults } \geq 65 \text { years } \\
(\mathrm{M}=86, \mathrm{SD}=5.7) \text { (age } \\
\text { range not given })\end{array}$ & $\begin{array}{l}\text { health (subjective), } \\
\text { self-efficacy }\end{array}$ & total PA & questionnaire: YPAS; DiPietro et al. [98] & health (subjective): +; self-efficacy: + \\
\hline
\end{tabular}


Table 1 (continued)

\begin{tabular}{|c|c|c|c|c|c|}
\hline $\begin{array}{l}\text { Reference } \\
\text { (first author) }\end{array}$ & Participants & $\begin{array}{l}\text { Individual charac- } \\
\text { teristics assessed }\end{array}$ & Type of PA & Measurement of PA & Results \\
\hline $\begin{array}{l}\text { Resnick } \\
{[99]}\end{array}$ & $\begin{array}{l}389 \text { older adults } \geq 65 \text { years } \\
\text { (sample } 1: M=82.5, S D= \\
6.9 ; \text { sample } 2: M=84, \\
S D=6.9 \text { ) (age range not } \\
\text { given) }\end{array}$ & $\begin{array}{l}\text { health (subjective), } \\
\text { self-efficacy }\end{array}$ & total PA & questionnaire: YPAS; DiPietro et al. [98] & health (subjective): 0 ; self-efficacy: + \\
\hline $\begin{array}{l}\text { Rowinski } \\
{[100]}\end{array}$ & $\begin{array}{l}4,813 \text { older adults } \geq 65 \\
\text { years (age range or other } \\
\text { statistics not given) }\end{array}$ & gender & $\begin{array}{l}\text { meeting PA } \\
\text { guidelines }\end{array}$ & $\begin{array}{l}\text { questionnaire: frequency of participation in different } \\
\text { moderate and vigorous activities over the last } 12 \text { months } \\
\text { (reference not provided) }\end{array}$ & gender: + \\
\hline $\begin{array}{l}\text { Schüz } \\
{[101]}\end{array}$ & $\begin{array}{l}309 \text { older adults } \geq 65 \text { years } \\
(\mathrm{M}=73.26, \mathrm{SD}=5.10) \\
\text { (age range not given) }\end{array}$ & $\begin{array}{l}\text { motivation } \\
\text { (intention) }\end{array}$ & total PA & questionnaire: IPAQ; Cerin et al. [36] & motivation: + \\
\hline $\begin{array}{l}\text { Shemesh } \\
{[102]}\end{array}$ & $\begin{array}{l}1,422 \text { older adults } 60-79 \\
\text { years (age mean or other } \\
\text { statistics not given) }\end{array}$ & $\begin{array}{l}\text { gender, health } \\
\text { (subjective) }\end{array}$ & total PA & $\begin{array}{l}\text { questionnaire: frequency of participation in different } \\
\text { activities (questionnaire available in the supplementary } \\
\text { material) }\end{array}$ & gender: 0 ; self-rated health: + \\
\hline $\begin{array}{l}\text { Simsek } \\
{[103]}\end{array}$ & $\begin{array}{l}2,947 \text { older adults } 65-106 \\
\text { years }(\mathrm{M}=72.2, \mathrm{SD}=5.6)\end{array}$ & education & total PA & questionnaire: IPAQ; Cerin et al. [36] & education: 0 \\
\hline $\begin{array}{l}\text { Siqueira } \\
{[104]}\end{array}$ & $\begin{array}{l}18,897 \text { adults, of these } \\
6,617 \text { older adults } \geq 60 \\
\text { years (age range or other } \\
\text { statistics not given) }\end{array}$ & gender, education & $\begin{array}{l}\text { meeting PA } \\
\text { guidelines }\end{array}$ & questionnaire: IPAQ; Cerin et al. [36] & gender: +; education: + \\
\hline $\begin{array}{l}\text { Sjögren } \\
{[105]}\end{array}$ & $\begin{array}{l}999 \text { older adults } 60-96 \\
\text { years }(M=74, S D \text { not } \\
\text { given })\end{array}$ & $\begin{array}{l}\text { education, marital } \\
\text { status }\end{array}$ & LTPA & $\begin{array}{l}\text { questionnaire: frequency of participation in different } \\
\text { moderate and vigorous activities in the last } 12 \text { months } \\
\text { (reference not provided) }\end{array}$ & education: 0 ; marital status: 0 \\
\hline $\begin{array}{l}\text { Snodgrass } \\
{[106]}\end{array}$ & $\begin{array}{l}200 \text { adults, of these } 87 \\
\text { older adults }>60 \text { years } \\
(\mathrm{M}=60.4, \mathrm{SD}=8.9)\end{array}$ & gender & total PA & accelerometer: ActiGraph GT3X worn on the hip & gender: 0 \\
\hline $\begin{array}{l}\text { Stephan } \\
{[107]}\end{array}$ & $\begin{array}{l}143 \text { older adults } 61-70 \\
\text { years }(M=64.5, S D=1.56)\end{array}$ & $\begin{array}{l}\text { gender, education, } \\
\text { marital status, } \\
\text { health (health } \\
\text { problems) }\end{array}$ & total PA & $\begin{array}{l}\text { questionnaire: frequency of participation in different } \\
\text { PAs during a week; question informed by Godin and } \\
\text { Shephard [108]; Vuillemin et al. [109] }\end{array}$ & $\begin{array}{l}\text { gender: -; education: } 0 \text {; marital } \\
\text { status: } 0 \text {; health (health problems): } 0\end{array}$ \\
\hline $\begin{array}{l}\text { Stuart } \\
{[110]}\end{array}$ & $\begin{array}{l}109 \text { older adults } 62-100 \\
\text { years (age mean or other } \\
\text { statistics not provided) }\end{array}$ & gender, education & total PA & $\begin{array}{l}\text { questionnaire: PA habits across the life span (frequency, } \\
\text { intensity, duration) (no reference provided) }\end{array}$ & gender: 0 ; education: + \\
\hline $\begin{array}{l}\text { Walsh } \\
{[111]}\end{array}$ & $\begin{array}{l}9,442 \text { older adults } \geq 65 \\
\text { years }(\mathrm{M}=71.7, \mathrm{SD}=5.3) \\
\text { (age range not given) }\end{array}$ & $\begin{array}{l}\text { education, health } \\
\text { (subjective) }\end{array}$ & $\begin{array}{l}\text { sports, total } \\
\text { PA }\end{array}$ & $\begin{array}{l}\text { questionnaire: modified Paffenbarger Scale; Vuillemin } \\
\text { et al. [109] }\end{array}$ & $\begin{array}{l}\text { education on sports: }+ \text {; education on } \\
\text { total PA: +; health (subjective) on } \\
\text { sports: } 0 \text {; health (subjective) on total } \\
\text { PA: + }\end{array}$ \\
\hline $\begin{array}{l}\text { Wister } \\
{[112]}\end{array}$ & $\begin{array}{l}11,630 \text { adults, of these } \\
2,126 \text { older adults } \geq 65 \\
\text { years (age range or other } \\
\text { statistics not provided) }\end{array}$ & $\begin{array}{l}\text { gender, education, } \\
\text { marital status, } \\
\text { employment }\end{array}$ & vigorous PA & questionnaire: Canada HPS; Statistics Canada [113] & $\begin{array}{l}\text { gender: +; education: +; marital } \\
\text { status: } 0 \text {; employment: - }\end{array}$ \\
\hline $\begin{array}{l}\text { Wolinsky } \\
{[114]}\end{array}$ & $\begin{array}{l}6,780 \text { older adults } \geq 70 \\
\text { years }(\mathrm{M}=76.46, \mathrm{SD}= \\
5.27) \text { (age range not given) }\end{array}$ & $\begin{array}{l}\text { gender, education, } \\
\text { health (health } \\
\text { problems, } \\
\text { subjective), locus } \\
\text { of control }\end{array}$ & walking & $\begin{array}{l}\text { questionnaire: walking a mile or more at least once a } \\
\text { week (part of the NHIS); Centers for Disease Control } \\
\text { and Prevention [115] }\end{array}$ & $\begin{array}{l}\text { gender: }+ \text {; education: }+ \text {; health } \\
\text { (health problems): } 0 \text {; health } \\
\text { (subjective): }+ \text {; locus of control: }+\end{array}$ \\
\hline $\begin{array}{l}\text { Yasunaga } \\
{[116]}\end{array}$ & $\begin{array}{l}3,084 \text { older adults } 65-99 \\
\text { years }\end{array}$ & gender & $\begin{array}{l}\text { sports, PA } \\
\text { in house/ } \\
\text { garden, } \\
\text { work-related } \\
\text { PA, PA for } \\
\text { transpor- } \\
\text { tation, total } \\
\text { PA }\end{array}$ & $\begin{array}{l}\text { accelerometer: electronic accelerometer (make and } \\
\text { model not specified) worn with a waist belt; } \\
\text { questionnaire: PAQ-EJ (available in the supplementary } \\
\text { material) }\end{array}$ & $\begin{array}{l}\text { accelerometer: gender on total PA: 0; } \\
\text { questionnaire: gender on sports: +; } \\
\text { gender on PA in house/garden: -; } \\
\text { gender on work-related PA: +; } \\
\text { gender on PA for transportation: + }\end{array}$ \\
\hline $\begin{array}{l}\text { Yusuf } \\
{[117]}\end{array}$ & $\begin{array}{l}7,801 \text { older adults } \geq 65 \\
\text { years (age range or other } \\
\text { statistics not given) }\end{array}$ & $\begin{array}{l}\text { education, health } \\
\text { (subjective) }\end{array}$ & LTPA & $\begin{array}{l}\text { questionnaire: Health Promotion and Disease } \\
\text { Prevention Supplement of the } 1990 \text { NHIS; Piani and } \\
\text { Schoenborn [118] }\end{array}$ & education: +; health (subjective): + \\
\hline $\begin{array}{l}\text { Zhao } \\
\text { [119] }\end{array}$ & $\begin{array}{l}99,172 \text { older adults } \geq 65 \\
\text { years }(M=74.3, \text { age range } \\
\text { and } S D \text { not given })\end{array}$ & $\begin{array}{l}\text { gender, education, } \\
\text { employment, } \\
\text { health (health } \\
\text { problems) }\end{array}$ & $\begin{array}{l}\text { meeting PA } \\
\text { guidelines }\end{array}$ & $\begin{array}{l}\text { questionnaire: participation in moderate and vigorous } \\
\text { activity in a week as assessed by the BRFSS survey; } \\
\text { Centers for Disease Control and Prevention [120] }\end{array}$ & $\begin{array}{l}\text { gender: +: education: +; employment: } \\
0 \text {; health (health problems): } 3 \text { times + }\end{array}$ \\
\hline
\end{tabular}

BRFSS, Behavioral Risk Factor Surveillance System; EPAQ2, EPIC-Norfolk Physical Activity Questionnaire; ERASS, Exercise, Recreation and Sport Survey; HPAQ, Habitual Physical Activity Questionnaire; HPS, Health Promotion Survey; IPAQ, International Physical Activity Questionnaire; LAPAQ, LASA Physical Activity Questionnaire; LTPA, leisuretime physical activity; NHANES, National Health and Nutrition Examination Survey; NHIS, National Health Interview Survey; PA, physical activity; PAQ, Physical Activity Questionnaire; PAQ-EJ, Physical Activity Questionnaire for Elderly Japanese; PASE, Physical Activity Scale for the Elderly; PASE-M, Physical Activity Scale for the Elderly translated into Malay language; QAPPA, Questionnaire d'Activité Physique pour les Personnes Âgées; WHO, World Health Organization; YPAS, Yale Physical Activity Survey. 


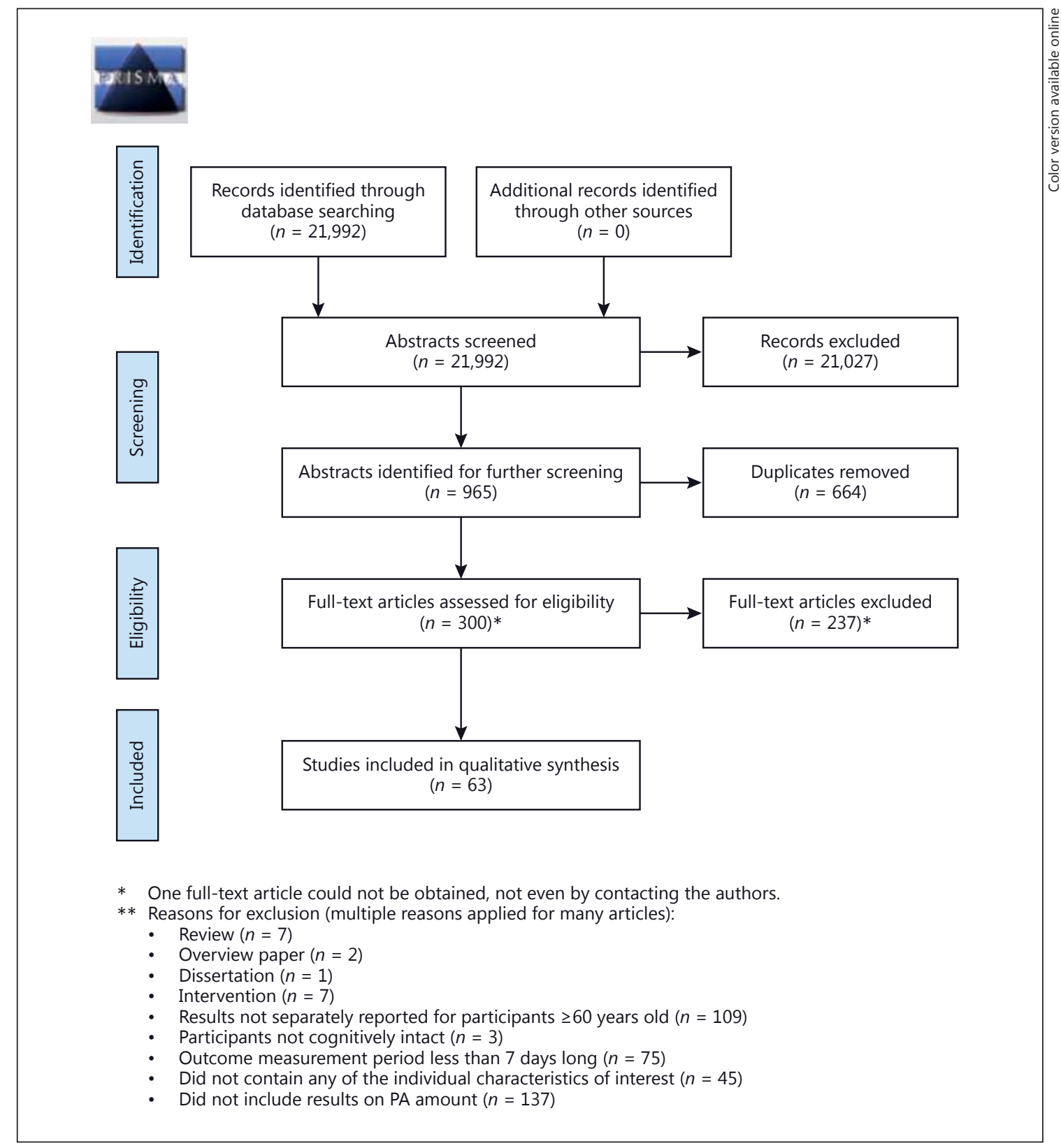

Fig. 1. Adapted PRISMA flow diagram illustrating the study selection process. PA, physical activity.

\section{Results of the Literature Search}

The study selection process is illustrated in Figure 1. We identified 21,992 records through database searching. We screened all 21,992 abstracts. Of these, 21,027 were excluded because they did not meet the eligibility criteria, resulting in 965 abstracts for further screening. After the removal of 664 duplicates, 301 articles were selected for full-text screening. One full-text could not be obtained, not even by contacting the authors; thus, we screened 300 full-text articles. Of these, 7 were excluded because they were review articles and would have led to duplicate inclusion of studies; 2 were overview papers and not quantitative studies; 1 was a dissertation that was not published in a peer-reviewed journal; 7 were intervention studies; 109 did not report results separately for participants aged at least 60 years; 3 included participants with cognitive impairments; in 75 , the measurement period 
for PA was shorter than 7 days; 45 did not examine the association between any of the individual characteristics of interest and PA; and 137 did not include information on the amount of PA participants engaged in. Notably, multiple reasons for exclusion applied for many articles. A total of 63 full-text articles were included in the review. Such a ratio of studies identified initially and eligible for inclusion in a review is common $[123,124]$.

The study characteristics are presented in Table 1. Most included studies examined the association between demographic variables and PA; only few considered psychological factors. Of the demographic variables, gender was examined most frequently, namely in 40 of the included studies, followed by education in 26 , marital status in 12 , and employment in 5 . Health was also examined in many included studies, subjective health in 21 and health problems in 14 . Of the psychological factors, self-efficacy was considered most frequently, namely in 7 included studies, followed by locus of control in 3 and motivation in 2. Life satisfaction was not examined in the included studies. PA was most frequently categorized as total PA (30 included studies), followed by meeting PA guidelines (11 studies), walking (9 studies), sports/structured exercise and leisure-time PA (7 studies for both), PA in house or garden (4 studies), work-related PA (1 study), PA for transportation (1 study), and vigorous PA (1 study). Light PA, moderate PA, and moderate and vigorous PA were not assessed in the included studies. Four studies measured PA with accelerometers only, 2 with accelerometers and questionnaires, and 59 relied on questionnaires only.

The risk of bias ratings for all studies included in the present paper are provided in Table 2. Considering all ten items, studies had between 2 and 9 "yes" ratings $(\mathrm{M}=$ $6.71, \mathrm{SD}=1.68, \mathrm{Md}=7.00$ ), between 0 and 4 "no" ratings $(\mathrm{M}=0.75, \mathrm{SD}=1.00, \mathrm{Md}=0.00)$, and between 1 and 5 "unclear" ratings $(\mathrm{M}=2.54, \mathrm{SD}=1.22, \mathrm{Md}=3.00)$. When "yes," "no," and "unclear" ratings were summed, total scores ranged from -2 to $9(\mathrm{M}=5.97, \mathrm{SD}=2.48)$.

\section{Results of the Data Extraction}

The results of the data extraction are presented in Table 3. For every pair of individual characteristic and PA outcome that was examined in the included studies, we recorded the number of positive associations, the number of negative associations, and the number of times when no association was found. For each individual characteristic, we also summed the number of positive, negative, and no associations across PA domains. Categorical vari-

Individual Characteristics and Physical Activity ables were treated in a dichotomous fashion, as described earlier.

\section{Gender}

Although possible gender differences in PA levels were examined in many studies, the results are somewhat inconclusive. Across all PA types, men were more active than women in 27 instances and less active in 7; no association between gender and PA level was found in 19 instances. Whether or not an association between gender and PA level can be observed may depend on the PA domain. Men had higher PA levels than women when vigorous PA, work-related PA, and PA for transportation (1 instance, respectively) were concerned, and in the majority of instances for the domains sports/exercise (5 instances versus 2 instances with no association) and leisure-time PA (3 instances versus 1 instance with no association). Women, on the other hand, performed more $\mathrm{PA}$ in house/garden (3 instances). The picture is somewhat less clear for walking and meeting PA guidelines, and particularly for total PA. Thus, to truly understand gender effects, it seems important to compare activitylevels between men and women within specific PA domains.

\section{Education}

Across all PA types, higher levels of education were associated with more PA participation in 21 instances, but in another 17, there was no association between education and PA. Only positive associations between education and PA level were found for the domains walking (1), PA in house/garden (1), and vigorous PA (1). More than half of the associations between education and PA level were positive for sports/exercise and meeting PA guidelines ( 4 positive associations versus 2 instances with no associations for both PA domains). For the pairing education and leisure-time PA, the majority of the associations were positive as well ( 6 versus 4 instances with no association). The picture looked different for total PA, where education was positively associated with PA in less than half (4) of the cases and not associated in 9 instances. Higher levels of education seem to be associated with more PA in domains in which PA is voluntary or something that individuals seek out. Further details on the activities included under total PA could help to further explain if this is the case.

\section{Marital Status}

Marital status was not associated with PA participation in most instances (11) across all PA categories; the ones considered included sports/exercise (2), walking (2),

Gerontology 2017;63:443-459

DOI: $10.1159 / 000475558$ 
Table 2. Risk of bias ratings

\begin{tabular}{|c|c|c|c|c|c|c|c|c|c|c|c|c|c|}
\hline $\begin{array}{l}\text { Reference } \\
\text { (first author) }\end{array}$ & $\begin{array}{l}\text { Adequate } \\
\text { sample } \\
\text { descrip- } \\
\text { tion? }\end{array}$ & $\begin{array}{l}\text { Sample } \\
\text { represen- } \\
\text { tative of } \\
\text { population } \\
\text { studied? }\end{array}$ & $\begin{array}{l}\text { Ratio- } \\
\text { nale for } \\
\text { sample } \\
\text { size? }\end{array}$ & $\begin{array}{l}\text { Use of } \\
\text { eligibility } \\
\text { criteria for } \\
\text { participant } \\
\text { selection? }\end{array}$ & $\begin{array}{l}\text { With- } \\
\text { drawals } \\
\text { reported } \\
\text { and } \\
\text { explained? }\end{array}$ & $\begin{array}{l}\text { Clear de- } \\
\text { scription of } \\
\text { measure- } \\
\text { ment instru- } \\
\text { ments? }\end{array}$ & $\begin{array}{l}\text { Use of } \\
\text { standard- } \\
\text { ized study } \\
\text { protocol? }\end{array}$ & $\begin{array}{l}\text { Appro- } \\
\text { priate } \\
\text { statistical } \\
\text { methods? }\end{array}$ & $\begin{array}{l}\text { Con- } \\
\text { founding } \\
\text { variables } \\
\text { controlled? }\end{array}$ & $\begin{array}{l}\text { Limita- } \\
\text { tions } \\
\text { acknowl- } \\
\text { edged? }\end{array}$ & $\begin{array}{l}\text { Yes } \\
\text { ratings }\end{array}$ & $\begin{array}{l}\text { No } \\
\text { ratings }\end{array}$ & $\begin{array}{l}\text { Unclear } \\
\text { ratings }\end{array}$ \\
\hline Arcury [33] & 1 & 1 & 1 & 1 & 0 & 1 & 1 & 1 & 1 & 1 & 9 & 0 & 1 \\
\hline Berger [34] & -1 & 1 & -1 & 1 & 0 & 1 & 1 & 1 & 1 & 1 & 7 & 2 & 1 \\
\hline Biernat [35] & 1 & 1 & -1 & 1 & 0 & 1 & 1 & 1 & 1 & 1 & 8 & 1 & 1 \\
\hline Bird [37] & 1 & 1 & 0 & 1 & 1 & 1 & 1 & 0 & 0 & 1 & 7 & 0 & 3 \\
\hline Black [38] & 1 & 1 & 0 & 1 & 1 & 1 & 1 & 1 & 1 & 1 & 9 & 0 & 1 \\
\hline Casado-Pérez [40] & 1 & 1 & 0 & 1 & 0 & 1 & 1 & 1 & 1 & 1 & 8 & 0 & 2 \\
\hline Chen $[41]$ & 1 & 1 & -1 & 1 & 1 & -1 & 1 & 0 & -1 & 1 & 6 & 3 & 1 \\
\hline Conn $[42]$ & 1 & 0 & -1 & 1 & -1 & 1 & 1 & 0 & 1 & 1 & 6 & 2 & 2 \\
\hline Danon-Hersch [44] & 0 & 1 & 0 & 1 & 1 & 1 & 1 & 1 & 1 & 1 & 8 & 0 & 2 \\
\hline de Souto Barreto [47] & 1 & 1 & 0 & 1 & 0 & 1 & 1 & 0 & 1 & 1 & 7 & 0 & 3 \\
\hline Egerton [49] & 1 & 1 & 0 & 1 & 1 & 1 & 1 & 1 & 1 & 1 & 9 & 0 & 1 \\
\hline Ferreira [50] & 1 & 0 & -1 & 1 & 1 & 1 & 1 & 1 & 1 & 1 & 8 & 1 & 1 \\
\hline Gao [51] & - & - & -1 & 1 & 1 & 1 & 1 & 1 & 1 & 1 & 7 & 1 & 2 \\
\hline Giuli [53] & 1 & 0 & 0 & 1 & 0 & 1 & 1 & 0 & 1 & 1 & 6 & 0 & 4 \\
\hline Grant-Savela [55] & 1 & 0 & 1 & 1 & 1 & 1 & 1 & 0 & -1 & 1 & 7 & 1 & 2 \\
\hline Grimby [57] & 0 & 0 & -1 & 0 & 0 & 1 & 1 & 1 & 1 & 1 & 5 & 1 & 4 \\
\hline Herbolsheimer [58] & 1 & 1 & 0 & 1 & 1 & 1 & 1 & 1 & 1 & 1 & 9 & 0 & 1 \\
\hline Hirakawa [59] & 0 & 0 & -1 & -1 & 1 & 0 & 1 & 0 & -1 & 1 & 3 & 3 & 4 \\
\hline Hirvensalo [60] & 0 & 1 & 0 & 1 & 1 & 1 & 1 & 0 & -1 & 1 & 6 & 1 & 3 \\
\hline Hughes [61] & 0 & 1 & 0 & 1 & 1 & 1 & 1 & 1 & 1 & 1 & 8 & 0 & 2 \\
\hline Huisingh-Scheetz [63] & 0 & 1 & 0 & 0 & 1 & 1 & 1 & 1 & 1 & 1 & 7 & 0 & 3 \\
\hline Ismail [64] & 1 & 0 & -1 & 1 & 0 & 1 & 1 & 0 & -1 & 1 & 5 & 2 & 3 \\
\hline Jerome [66] & 0 & 0 & 0 & 1 & 1 & 1 & 1 & 1 & 1 & 1 & 7 & 0 & 3 \\
\hline Johansson [69] & 1 & 1 & -1 & 1 & 0 & 1 & 1 & 1 & 1 & 1 & 8 & 1 & 1 \\
\hline Kahana [70] & 1 & 0 & -1 & 0 & 0 & 1 & 1 & 1 & 1 & -1 & 5 & 2 & 3 \\
\hline Kaur [71] & 0 & 1 & 0 & 0 & 1 & 1 & 1 & 1 & 0 & 1 & 6 & 0 & 4 \\
\hline Kendig [73] & 0 & 1 & 1 & 1 & 1 & 1 & 1 & 1 & 1 & 1 & 9 & 0 & 1 \\
\hline Kerr [75] & 0 & 0 & 0 & 0 & 1 & 1 & 1 & 1 & 1 & 1 & 6 & 0 & 4 \\
\hline Lawlor [76] & 0 & 0 & 0 & 0 & 1 & 1 & 1 & 1 & 1 & 1 & 6 & 0 & 4 \\
\hline Lee [77] & 1 & 0 & -1 & 1 & 0 & 1 & 1 & 1 & -1 & 1 & 6 & 2 & 2 \\
\hline Lee [79] & 1 & 0 & 1 & 1 & 1 & 1 & 1 & 1 & 1 & 1 & 9 & 0 & 1 \\
\hline Leveille [80] & 0 & 0 & -1 & 0 & 1 & 1 & 1 & 1 & 0 & 1 & 5 & 1 & 4 \\
\hline Lian [81] & 0 & 1 & 0 & 0 & 0 & 1 & 1 & 1 & 1 & 1 & 6 & 0 & 4 \\
\hline $\operatorname{Lim}[82]$ & 0 & 1 & 0 & 1 & 0 & 1 & 1 & 1 & 1 & 1 & 7 & 0 & 3 \\
\hline Loland [83] & 1 & 1 & 0 & 0 & 0 & 1 & 1 & 0 & -1 & -1 & 4 & 2 & 4 \\
\hline Mäkilä [84] & 0 & 1 & 0 & 1 & 1 & 1 & 1 & 0 & -1 & 0 & 5 & 1 & 4 \\
\hline Menec [85] & 1 & 1 & 0 & 1 & 1 & 1 & 1 & 1 & 1 & 1 & 9 & 0 & 1 \\
\hline Merom [86] & 0 & 1 & 0 & 1 & 0 & 1 & 1 & 1 & 1 & 1 & 7 & 0 & 3 \\
\hline Mier [88] & 0 & 0 & 0 & 1 & 1 & 1 & 1 & 1 & 1 & 1 & 7 & 0 & 3 \\
\hline Moschny [89] & 1 & 0 & -1 & 1 & 1 & 1 & 1 & 1 & 0 & 1 & 7 & 1 & 2 \\
\hline Murphy [91] & 0 & 1 & 0 & 1 & 0 & 1 & 1 & 1 & 0 & 1 & 6 & 0 & 4 \\
\hline Mynarski [92] & -1 & 0 & -1 & 0 & 0 & 1 & 1 & 0 & -1 & 0 & 2 & 3 & 5 \\
\hline Palacios-Ceña [93] & 0 & 1 & 0 & 1 & 0 & 1 & 1 & 1 & 1 & 1 & 7 & 0 & 3 \\
\hline Persson [94] & 1 & -1 & -1 & 0 & 0 & 1 & 1 & 1 & 1 & 1 & 6 & 2 & 2 \\
\hline Reigal [95] & 1 & 0 & -1 & 0 & 0 & -1 & 1 & 0 & -1 & -1 & 2 & 4 & 4 \\
\hline Resnick [96] & 1 & 0 & -1 & 1 & 0 & 0 & 1 & 1 & 1 & 1 & 6 & 1 & 3 \\
\hline Resnick [97] & 1 & 0 & -1 & 1 & 1 & 1 & 1 & 1 & 1 & 1 & 8 & 1 & 1 \\
\hline Resnick [99] & 1 & 1 & 0 & 1 & 1 & 1 & 1 & 1 & 1 & 1 & 9 & 0 & 1 \\
\hline Rowinski [100] & 0 & 1 & 1 & 0 & 0 & 1 & 1 & 0 & -1 & 1 & 5 & 1 & 4 \\
\hline Schüz [101] & 1 & 1 & 0 & 1 & 1 & 1 & 1 & 1 & 1 & 1 & 9 & 0 & 1 \\
\hline Shemesh [102] & 0 & 1 & -1 & -1 & 0 & 1 & 1 & 0 & -1 & 1 & 4 & 3 & 3 \\
\hline Simsek [103] & 1 & 0 & 1 & 1 & 0 & 1 & 1 & 1 & 1 & 1 & 8 & 0 & 2 \\
\hline Siqueira [104] & -1 & 1 & 0 & 0 & 1 & 1 & 1 & 1 & 1 & 1 & 7 & 1 & 2 \\
\hline Sjögren [105] & 1 & 1 & 0 & 1 & 1 & 1 & 1 & 1 & 1 & 1 & 9 & 0 & 1 \\
\hline Snodgrass [106] & 1 & 1 & 0 & 0 & 1 & 1 & 1 & 1 & 1 & 1 & 8 & 0 & 2 \\
\hline Stephan [107] & 1 & 0 & -1 & 1 & 1 & 1 & 1 & 1 & 1 & 1 & 8 & 1 & 1 \\
\hline Stuart [110] & 0 & 0 & -1 & 0 & 1 & 0 & 1 & 1 & 1 & 1 & 5 & 1 & 4 \\
\hline Walsh [111] & 1 & 0 & -1 & 1 & 0 & 1 & 1 & 1 & 1 & 1 & 7 & 1 & 2 \\
\hline Wister [112] & 0 & 0 & 0 & 0 & 0 & 1 & 1 & 1 & 1 & 1 & 5 & 0 & 5 \\
\hline Wolinsky [114] & 1 & 0 & 0 & 1 & 0 & 0 & 1 & 1 & 1 & 1 & 6 & 0 & 4 \\
\hline Yasunaga [116] & 1 & 1 & 0 & 1 & 1 & 1 & 1 & 0 & 1 & 1 & 8 & 0 & 2 \\
\hline Yusuf [117] & 0 & 1 & 0 & 0 & 1 & 1 & 1 & 1 & 1 & 1 & 7 & 0 & 3 \\
\hline Zhao [119] & 1 & 1 & 0 & 0 & 0 & 1 & 1 & 1 & 1 & 1 & - & 0 & 3 \\
\hline Total yes scores & 35 & 27 & 23 & 42 & 34 & 57 & 63 & 47 & 46 & 58 & - & - & - \\
\hline Total no scores & 3 & 1 & 34 & 2 & 1 & 2 & 0 & 0 & 12 & 3 & - & - & - \\
\hline Total unclear scores & 25 & 35 & 6 & 19 & 28 & 4 & 0 & 16 & 5 & 2 & - & - & - \\
\hline
\end{tabular}


Table 3. Results of the data extraction

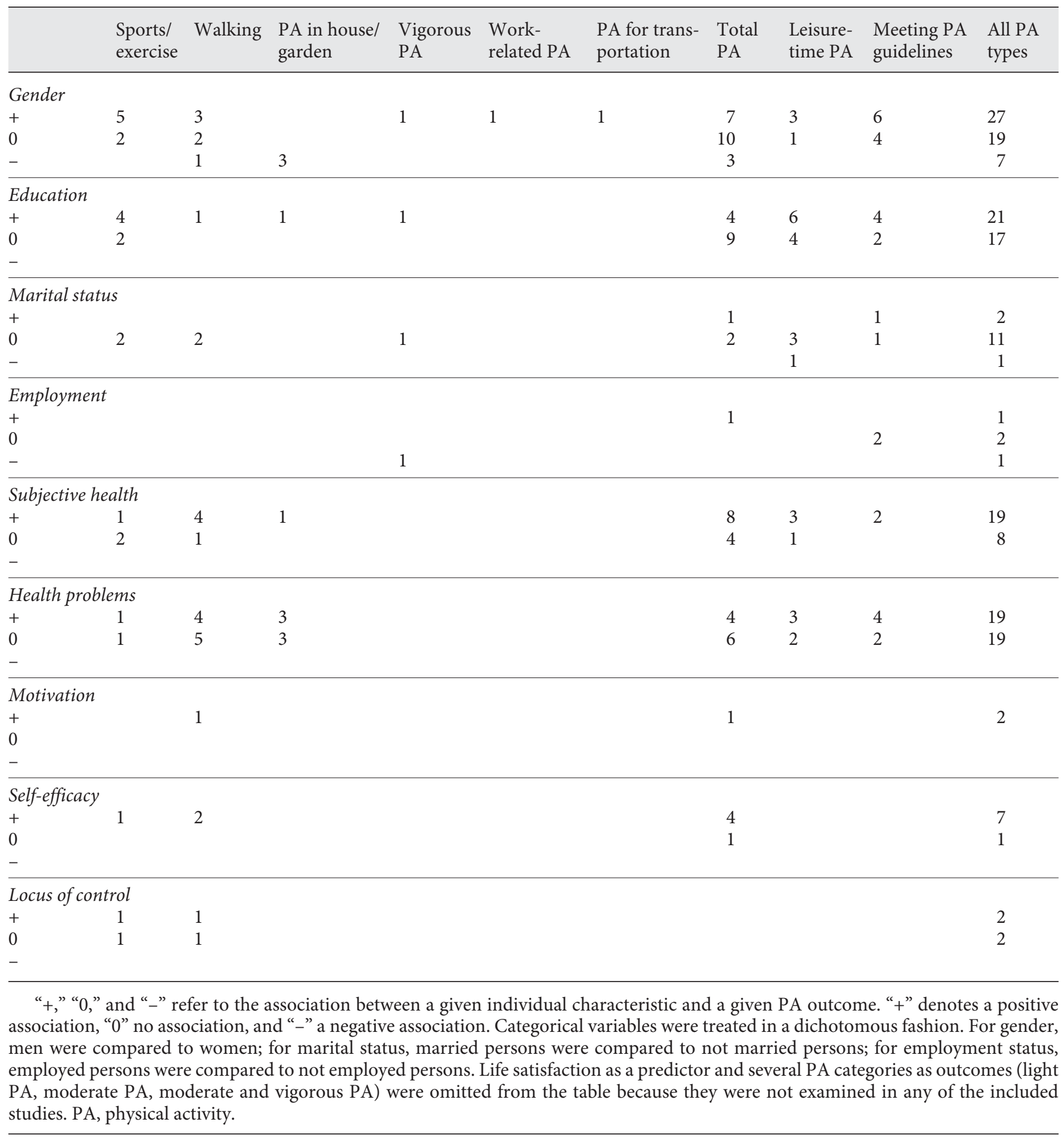

Individual Characteristics and Physical Activity 
vigorous PA (1), total PA (2), leisure-time PA (3), and meeting PA guidelines (1). In 1 instance (total PA), married older adults had higher PA levels than those who were not married, and in 1 instance (leisure-time PA), married older adults had lower PA levels than those who were not married.

\section{Employment}

Employment status does not seem to have a strong association with PA levels. No association was found with meeting PA guidelines (2 instances). Employment status was positively associated with total PA once and negatively associated with vigorous PA once.

\section{Subjective Health}

In most instances (19), a positive association was observed between subjective health and PA levels when all domains were considered together. For the outcomes PA in house/garden and meeting PA guidelines, only positive associations with subjective health were found, but these pairings were examined in very few studies ( 1 and 2, respectively). For walking, total PA, and leisure-time PA, a positive association with subjective health was found in the majority of instances, but for sports/exercise only one-third of the time. Overall, no association between subjective health and PA was found in 8 instances; these referred to the domains sports/exercise (2), walking (1), total PA (4), and leisure-time PA (1).

\section{Health Problems}

The role of health problems (presence of a chronic condition or number of health problems) for PA engagement is less clear than that of subjective health. Across all PA domains, fewer health problems or the absence of a chronic condition were associated with higher PA levels in 19 instances, but in another 19, no association was found. Reading of the included studies suggested that chronic conditions differ in whether or not they affect PA participation or which PA domains they affect. However, the data were insufficient to make formal comparisons.

\section{Motivation}

Higher levels of motivation were associated with more PA across the two examined PA domains walking ( 1 instance) and total PA (1 instance). Motivation was measured as reasons that motivated participants to walk in the past week in the study focusing on walking and as intention in the study with total PA as an outcome.

\section{Self-Efficacy}

Higher self-efficacy was associated with more PA across domains in most instances (7). Of these, 1 referred to the association with sports/exercise, 2 to walking, and 4 to total PA. Only in 1 instance was self-efficacy not associated with PA level; this was the case for total PA.

\section{Locus of Control}

An external locus of control was associated with more PA in 2 instances across PA domains, 1 referring to sports/ exercise and 1 to walking. However, in another 2 also referring to sports/exercise and walking, there was no association between locus of control and walking. Reading of the included studies suggested that the presence or absence of an association may depend on the type of control measure used (e.g., general locus of control versus health locus of control), but the data were too sparse to allow for formal comparisons.

\section{Discussion}

The goal of this systematic review was to identify individual characteristics that are consistently linked to higher PA levels in older adults. Specifically, we set out to answer the question of how specific individual characteristics (gender, education, marital status, employment, physical health, motivation, self-efficacy, life satisfaction, and locus of control) of cognitively intact older adults are related to routine PA in their daily lives. We applied an innovative approach by considering various types of PA separately. One main finding of this review was that only a relatively small number of the included studies had measured PA in one of these domains or specified clearly in which domain PA had been measured. For the vast majority, the outcome PA had to be listed in the "total PA" category.

Although few included studies examined associations between the psychological factors (motivation, self-efficacy, locus of control) and PA, the picture is clearest for this group of variables. Of course, given the small number of studies linking psychological factors and routine PA in older adults, the results have to be interpreted with caution. Across PA domains, higher levels of motivation and self-efficacy were associated with more PA in almost all instances. Findings regarding the association between locus of control and PA are inconclusive, possibly because the operationalization of control differed between studies. In the future, a differentiation between control domains could clarify these results. 
The picture regarding the association between individual characteristics and PA levels is less clear for the demographic variables, although they were examined in many of the included studies. Considering all types of PA together, it remained relatively unclear whether there are gender differences in activity levels. It seemed that overall PA levels may not differ between men and women. However, men may be more active than women in some domains (e.g., vigorous PA, leisure-time PA), whereas women may be more active than men in others (e.g., PA in house/garden). Some of these results may be due to today's older adults' still adopting relatively traditional gender roles, and patterns may change as new cohorts reach old age. The effects of education also seem to depend on domain. Higher levels of education may be associated with higher PA levels whenever individuals specifically need to seek out PA (e.g., sports/exercise) or when knowledge is relevant (e.g., meeting PA guidelines). Further specification of activities in studies currently labeled as total PA could clarify this idea. Marital and employment status, on the other hand, seem to contribute little to explaining individual differences in PA levels of older adults.

With regards to health, it may be more important how someone is feeling subjectively than whether or not he or she suffers from one or more health problems. We observed that better subjective health was associated with more PA in the majority of cases and across PA domains. Health problems, even chronic ones, do not necessarily prevent older adults from being active. In this review, we saw that in some cases, they even resulted in greater PA participation, whereas in others, they had no effect. This may also depend on PA domain.

\section{Strengths, Limitations, and Future Directions}

One of the main strengths of this review was its application of the broad definition of PA by Caspersen et al. [14] and the differentiation of the outcome PA by domains. However, in many of the included studies, the outcome had to be categorized as total PA because the studies did not differentiate between domains or lacked specific details on the context in which PA was measured. It is possible that this contributed to the inconclusive results concerning the association of several variables and PA. We also included studies applying objective and self-report measures of PA. Due to the low number of studies that measured PA objectively, it was not possible to compare whether effects differed by measurement method. Furthermore, the self-report measures utilized in the included studies differed; it is thus possible that inconsistencies in associations between individual characteristics

Individual Characteristics and Physical Activity and PA are - to some degree - due to differences in measurement instruments. One issue of studies with objective PA measures was that the majority did not differentiate PA by domains and only reported total PA.

We took a comprehensive look at the association between individual characteristics and PA by considering characteristics from three domains: demographic variables, health, and psychological factors. Our findings suggest that psychological factors are relatively reliably associated with PA, although this conclusion should be viewed with some caution because only a relatively small number of included studies examined psychological variables. Two of the demographic variables, marital and employment status, on the other hand, seemed to have little relevance for PA. It is possible that their effects were somewhat obscured by the dichotomous coding (married versus not married, employed versus not employed) that we applied in order to be able to utilize the same data extraction method as for the other variables; in the future, it would be useful to consider other levels of these variables.

Our quantitative data extraction approach went beyond a simple narrative summary. A future meta-analytic approach taking into consideration effect sizes would be a useful next step. It has the potential to shed more light onto some of the inconclusive results. Additionally, effect sizes could lead to further insights into how influential the different individual characteristics are relative to each other. With meta-analytic techniques, it would be possible to examine associations of several individual characteristics with PA levels simultaneously. Furthermore, one could account for heterogeneity in associations due to the different measurement instruments that were used in the included studies, and determine to what extent study quality is linked to the likelihood of reporting a significant result.

\section{Implications}

The findings from this review have a number of important theoretical and practical implications. Individual characteristics seem to be differentially associated with PA levels in distinct domains; thus, explicit measurement and promotion of PA in various domains seems necessary. In intervention studies and practical settings, it may make sense to target those activities that people may be predisposed to engage in based on their individual characteristics, or specifically those that they may engage in to a lesser degree. A clear description of PA measurement instruments would facilitate a better comparison of study results as well as conclusions regarding whether participants are close to meeting the WHO PA guidelines.

Gerontology 2017;63:443-459

DOI: $10.1159 / 000475558$ 


\section{Conclusion}

In this systematic review, we observed that two psychological factors - motivation and self-efficacy - and the perception of one's health seem to be consistently linked to higher PA levels in older adults in a small number of studies that met the inclusion criteria. Selected demographic variables - gender and education - may be important for some types of PA. Perhaps most importantly, this review suggests that differentiation of PA by domains is crucial for identifying and understanding which individual characteristics are associated with PA levels and how.

\section{Acknowledgment}

This work was funded by grant H2020-MSCA-IF-2014 661555 from the European Commission.

\section{References}

1 American College of Sports Medicine, Chodzko-Zajko WJ, Proctor DN, Fiatarone Singh MA, Minson CT, Nigg CR, Salem GJ, Skinner JS: American College of Sports Medicine position stand. Exercise and physical activity for older adults. Med Sci Sports Exerc 2009;41: 1510-1530.

2 O’Donovan G, Blazevich AJ, Boreham C, Cooper AR, Crank H, Ekelund U, Fox KR, Gately P, Giles-Corti B, Gill JMR, Hamer M, McDermott I, Murphy M, Mutrie N, Reilly JJ, Saxton JM, Stamatakis E: The ABC of Physical Activity for Health: a consensus statement from the British Association of Sport and Exercise Sciences. J Sports Sci 2010;28:573-591.

3 Langsetmo L, Hitchcock C, Kingwell EJ, Davison KS, Berger C, Forsmo S, Zhou W, Kreiger N, Prior JC: Physical activity, body mass index and bone mineral density - associations in a prospective population-based cohort of women and men: the Canadian Multicentre Osteoporosis Study (CaMos). Bone 2012;50:401-408.

4 Colcombe SJ, Kramer AF: Fitness effects on the cognitive function of older adults: a metaanalytic study. Psychol Sci 2003;14:125-130.

5 Withall J, Stathi A, Davis M, Coulson J, Thompson JL, Fox KR: Objective physical activity and sedentary time and associations with subjective well-being in adults aged 70 and over. Int J Environ Res Public Health 2014;11:643-656.

6 Studenski S, Faulkner K, Inzitari M, Brach J, Chandler J, Cawthon P, Connor EB, Nevitt M, Visser M, Kritchevsky S, Badinelli S, Harris T, Newman AB, Cauley J, Ferrucci L, Guralnik J: Gait speed and survival in older adults. JAMA 2011;305:50-58.

7 Lee IM, Shiroma EJ, Lobelo F, Puska P, Blair SN, Katzmarzyk PT; Lancet Physical Activity Series Working Group: Effect of physical inactivity on major non-communicable diseases worldwide: an analysis of burden of disease and life expectancy. Lancet 2012;380:219-229.

8 Caspersen CJ, Powell KE, Christenson GM: Physical activity, exercise, and physical fitness: definitions and distinctions for healthrelated research. Public Health Rep 1985;100: 126-131.
9 Morris JN, Hardman AE: Walking to health. Sports Med 1997;23:306-332.

10 Marshall SJ, Levy SS, Tudor-Locke CE, Kolkhorst FW, Wooten KM, Ji M, Macera CA, Ainsworth BE: Translating physical activity recommendations into a pedometer-based step goal: 3000 steps in 30 minutes. Am J Prev Med 2009;36:410-415.

11 World Health Organization: Global recommendations on physical activity for health. 2010. http://apps.who.int/iris/bitstream/ 10665/44399/1/9789241599979_eng.pdf.

12 Sun F, Norman IJ, While AE: Physical activity in older people: a systematic review. BMC Public Health 2013;13:449.

13 Hallal PC, Andersen LB, Bull FC, Guthold R, Haskell W, Ekelund U; Lancet Physical Activity Series Working Group: Global physical activity levels: surveillance progress, pitfalls, and prospects. Lancet 2012;380:247-257.

14 European Commission: Special Eurobarometer 412: Sport and Physical Activity. 2014. http://ec.europa.eu/public_opinion/archives/ebs/ebs_412_en.pdf.

15 Centers for Disease Control and Prevention: Vital signs: walking among adults - United States, 2005 and 2010. MMWR Morb Mortal Wkly Rep 2012;61:595-601.

16 Sallis JF, Owen N: Physical Activity and Behavioral Medicine. Thousand Oaks, Sage Publications, 1999, pp 110-134.

17 Trost SG, Owen N, Bauman AE, Sallis JF, Brown W: Correlates of adults' participation in physical activity: review and update. Med Sci Sports Exerc 2002;34:1996-2001.

18 Peclová J, Frömel K, Cuberek R: Gender-specific associations between perceived neighborhood walkability and meeting walking recommendations when walking for transport and recreation for Czech inhabitants over 50 years of age. Int J Environ Res Publ Health 2014;11:527-536.

19 Martin KA, Sinden AR: Who will stay and who will go? A review of older adults' adherence to randomized controlled trials of exercise. J Aging Phys Act 2001;9:91-114.
20 Van Stralen MM, de Vries H, Mudde AN, Bolman C, Lechner L: Determinants of initiation and maintenance of physical activity among older adults: a literature review. Health Psychol Rev 2009;3:147-207.

21 Koeneman MA, Verheijden MW, Chinapaw MJ, Hopman-Rock M: Determinants of physical activity and exercise in healthy older adults: a systematic review. Int J Behav Nutr Phys Act 2011;8:142.

22 Ajzen I: The theory of planned behavior. Organ Behav Hum Decis Process 1991;50:179211.

23 Deci EL, Ryan RM: Self-determination theory: a macrotheory of human motivation, development, and health. Can Psychol 2008;49: 182.

24 Bandura A: Social cognitive theory: an agentic perspective. Annu Rev Psychol 2001;52:1-26.

25 Moher D, Liberati A, Tetzlaff J, Altman DG; PRISMA Group: Preferred reporting items for systematic reviews and meta-analyses: the PRISMA statement. Ann Intern Med 2009; 151:264-269, W64.

26 United Nations: World Population Ageing. 2015. http://www.un.org/en/development/ desa/population/publications/pdf/ageing/ WPA2015_Report.pdf.

27 Baranowski T, Masse LC, Ragan B, Welk G: How many days was that? We're still not sure, but we're asking the question better! Med Sci Sports Exerc 2008;40(7 suppl):S544-S549.

28 Matthews CE, Hagströmer M, Pober DM, Bowles HR: Best practices for using physical activity monitors in population-based research. Med Sci Sports Exerc 2012;44(1 suppl 1):S68-S76.

29 Ainsworth BE, Caspersen CJ, Matthews CE, Mâsse LC, Baranowski T, Zhu W: Recommendations to improve the accuracy of estimates of physical activity derived from self report. J Phys Act Health 2012;9(suppl 1): S76-S84.

30 Landis JR, Koch GG: The measurement of observer agreement for categorical data. Biometrics 1977;33:159-174.

31 McHugh ML: Interrater reliability: the kappa statistic. Biochem Med (Zagreb) 2012;22: 276-282. 
32 APA Publications and Communications Board Working Group on Journal Article Reporting Standards: Reporting standards for research in psychology: Why do we need them? What might they be? Am Psychol 2008; 63:839-851.

33 Arcury TA, Snively BM, Bell RA, Smith SL, Stafford JM, Wetmore-Arkader LK, Quandt SA: Physical activity among rural older adults with diabetes. J Rural Health 2006;22:164-168.

34 Berger U, Der G, Mutrie N, Hannah MK: The impact of retirement on physical activity. Ageing Soc 2005;25:181-195.

35 Biernat E, Tomaszewski P: Socio-demographic and leisure activity determinants of physical activity of working Warsaw residents aged 60 to 69 years. J Hum Kinet 2011;30: 173-181.

36 Cerin E, Cain KL, Oyeyemi A, Owen N, Conway TL, Cochrane T, van Dyck D, Schipperijn J, Mitaš J, Toftager M, Aguinaga-Ontoso I, Sallis JF: Correlates of agreement between accelerometry and self-reported physical activity. Med Sci Sports Exerc 2016;48:1075-1084.

37 Bird SR, Radermacher H, Sims J, Feldman S, Browning C, Thomas S: Factors affecting walking activity of older people from culturally diverse groups: an Australian experience. J Sci Med Sport 2010;13:417-423.

38 Black SV, Cooper R, Martin KR, Brage S, Kuh D, Stafford M: Physical activity and mental well-being in a cohort aged 60-64 years. Am J Prev Med 2015;49:172-180.

39 Wareham NJ, Jakes RW, Rennie KL, Mitchell J, Hennings S, Day NE: Validity and repeatability of the EPIC-Norfolk Physical Activity Questionnaire. Int J Epidemiol 2002;31:168-174.

40 Casado-Pérez C, Hernández-Barrera V, Jiménez-García R, Fernández-de-las-Peñas $\mathrm{C}$, Carrasco-Garrido P, López-de-Andrés A, Palacios-Ceña $\mathrm{D}$ : Time trends in leisure time physical activity and physical fitness in the elderly: five-year follow-up of the Spanish National Health Survey (2006-2011). Maturitas 2015;80:391-398.

41 Chen KM, Lin MH, Wang YC, Huang HT, Li $\mathrm{CH}$ : A model-based survey of physical health in community-dwelling older adults. J Nurs Res 2012;20:239-248.

42 Conn VS, Burks KJ, Pomeroy SL, Cochran JE: Are there different predictors of distinct exercise components? Rehabil Nurs 2003;28:8791, 97.

43 Baecke J, Burema J, Frijters J: A short questionnaire for the measurement of habitual physical activity in epidemiological studies. Am J Clin Nutr 1982;36:936-942.

44 Danon-Hersch N, Santos-Eggimann B: Physical activity in daily life is associated with lower adiposity values than doing weekly sports in Lc65+ cohort at baseline. BMC Public Health 2013;13:1175.

45 Sequeira MM, Rickenbach M, Wietlisbach V, Tullen B, Schutz Y: Physical activity assessment using a pedometer and its comparison with a questionnaire in a large population survey. Am J Epidemiol 1995;142:989-999.
46 Wietlisbach V, Paccaud F, Rickenbach M, Gutzwiller F: Trends in cardiovascular risk factors (1984-1993) in a Swiss region: results of three population surveys. Prev Med 1997; 26:523-533.

47 de Souto Barreto P: Construct and convergent validity and repeatability of the Questionnaire d'Activité Physique pour les Personnes Âgées (QAPPA), a physical activity questionnaire for the elderly. Public Health 2013;127: 844-853.

48 Barreto PS, Ferrandez AM, Saliba-Serre B: Validation of the QAPPA, a new tool for assessing physical activity among Frenchspeaking elderly. Sci Sports 2011;26:11-18.

49 Egerton T, Chastin SF, Stensvold D, Helbostad JL: Fatigue may contribute to reduced physical activity among older people: an observational study. J Gerontol A Biol Sci Med Sci 2016;71:670-676.

50 Ferreira MT, Matsudo SM, Ribeiro MC, Ramos LR: Health-related factors correlate with behavior trends in physical activity level in old age: longitudinal results from a population in São Paulo, Brazil. BMC Public Health 2010; 10:690.

51 Gao J, Fu H, Li J, Jia Y: Association between social and built environments and leisuretime physical activity among Chinese older adults - a multilevel analysis. BMC Public Health 2015;15:1317.

52 Macfarlane D, Chan A, Cerin E: Examining the validity and reliability of the Chinese version of the International Physical Activity Questionnaire, long form (IPAQ-LC). Public Health Nutr 2011;14:443-450.

53 Giuli C, Papa R, Mocchegiani E, Marcellini F: Predictors of participation in physical activity for community-dwelling elderly Italians. Arch Gerontol Geriatr 2012;54:50-54.

54 Marcellini F, Giuli C, Papa R, Gagliardi C, Malavolta M, Mocchegiani E: BMI, life-style and psychological conditions in a sample of elderly Italian men and women. J Nutr Health Aging 2010;14:515-522.

55 Grant-Savela SD: Active living among older residents of a rural naturally occurring retirement community. J Appl Gerontol 2010;29: 531-553.

56 New England Research Institutes: PASE physical activity scale for the elderly: administration and scoring instruction manual. Watertown, New England Research Institutes, 1991.

57 Grimby A, Johansson AK, Sundh V, Grimby G: Walking habits in elderly widows. Am J Hosp Palliat Care 2008;25:81-87.

58 Herbolsheimer F, Schaap LA, Edwards MH, Maggi S, Otero Á, Timmermans EJ, Denkinger MD, van der Pas S, Dekker J, Cooper C, Dennison EM, van Schoor NM, Peter R; Eposa Study Group: Physical activity patterns among older adults with and without knee osteoarthritis in six European countries. Arthritis Care Res (Hoboken) 2016;68:228-236.
59 Hirakawa Y, Kimata T, Uemura K: Factors associated with self-rated health in the rural population: age- and gender-specific analysis. J Rural Med 2013;82:222-227.

60 Hirvensalo M, Lampinen P, Rantanen T: Physical exercise in old age: an eight-year follow-up study on involvement, motives, and obstacles among persons age 65-84. J Aging Phys Act 1998;6:157-168.

61 Hughes JP, McDowell MA, Brody DJ: Leisure-time physical activity among US adults 60 or more years of age: results from NHANES 1999-2004. J Phys Act Health 2008;5:347358.

62 Centers for Disease Control and Prevention: National Health and Nutrition Examination Survey. http://www.cdc.gov/nchs/about/major/nhanes/nhanes2003-2004/questexamO3_O4.htm.

63 Huisingh-Scheetz M, Kocherginsky M, Schumm PL, Engelman M, McClintock MK, Dale W, Waite L: Geriatric syndromes and functional status in NSHAP: rationale, measurement, and preliminary findings. J Gerontol B Psychol Sci Soc Sci 2014;69(suppl 2): S177-S190.

64 Ismail N, Hairi F, Choo WY, Hairi NN, Peramalah D, Bulgiba A: The Physical Activity Scale for the Elderly (PASE): validity and reliability among community-dwelling older adults in Malaysia. Asia Pac J Public Health 2015;27:62-72.

65 Washburn RA, Smith KW, Jette AM, Janney CA: The Physical Activity Scale for the Elderly (PASE): development and evaluation. J Clin Epidemiol 1993;46:153-162.

66 Jerome GJ, Glass TA, Mielke M, Xue QL, Andersen RE, Fried LP: Physical activity participation by presence and type of functional deficits in older women: the Women's Health and Aging Studies. J Gerontol A Biol Sci Med Sci 2006;61:1171-1176.

67 Folsom AR, Jacobs DR Jr, Caspersen CJ, Gomez-Marin O, Knudsen J: Test-retest reliability of the Minnesota Leisure Time Physical Activity Questionnaire. J Chronic Dis 1986; 39:505-511.

68 Taylor HL, Jacobs DR Jr, Schucker B, Knudsen J, Leon AS, Debacker G: A questionnaire for the assessment of leisure time physical activities. J Chronic Dis 1978;31:741-755

69 Johansson J, Nordström A, Nordström P: Greater fall risk in elderly women than in men is associated with increased gait variability during multitasking. J Am Med Dir Assoc 2016;17:535-540.

70 Kahana E, Kahana B, Zhang J: Motivational antecedents of preventive proactivity in late life: linking future orientation and exercise. Motiv Emot 2005;29:443-464.

71 Kaur J, Kaur G, Ho BK, Yao WK, Salleh M, Lim KH: Predictors of physical inactivity among elderly Malaysians: recommendations for policy planning. Asia Pac J Public Health 2015;27:314-322. 
72 World Health Organization: The WHO STEPwise Approach to Noncommunicable Disease Risk Factor Surveillance (STEPS). 2016. http://www.who.int/chp/steps/instrument/STEPS_Instrument_V3.1.pdf?ua $=1$.

73 Kendig H, Browning C, Teshuva K: Health actions and social class among older Australians. Aust N Z J Public Health 1998;22:808813.

74 Risk Factor Prevalence Management Committee: Risk-Factor Prevalence Study. Canberra, National Heart Foundation of Australia, 1990.

75 Kerr J, Sallis JF, Saelens BE, Cain KL, Conway TL, Frank LD, King AC: Outdoor physical activity and self rated health in older adults living in two regions of the U.S. Int J Behav Nutr Phys Act 2012;9:89.

76 Lawlor DA, Taylor M, Bedford C, Ebrahim S: Is housework good for health? Levels of physical activity and factors associated with activity in elderly women. Results from the British Women's Heart and Health Study. J Epidemiol Community Health 2002;56:473-478.

77 Lee YS: Gender differences in physical activity and walking among older adults. J Women Aging 2005; 17:55-70.

78 Voorrips LE, Ravelli AC, Dongelmans PC, Deurenberg P, Van Staveren WA: A physical activity questionnaire for the elderly. Med Sci Sports Exerc 1991;23:974-979.

79 Lee YS, Laffrey SC: Predictors of physical activity in older adults with borderline hypertension. Nurs Res 2006;55:110-120.

80 Leveille SG, Cohen-Mansfield J, Guralnik JM: The impact of chronic musculoskeletal pain on exercise attitudes, self-efficacy, and physical activity. J Aging Phys Act 2003;11:275283.

81 Lian WM, Gan GL, Pin CH, Wee S, Ye HC: Correlates of leisure-time physical activity in an elderly population in Singapore. Am J Public Health 1999;89:1578-1580.

82 Lim K, Taylor L: Factors associated with physical activity among older people - a population-based study. Prev Med 2005;40:33-40.

83 Loland NW: Exercise, health, and aging. J Aging Phys Act 2004;12:170-184.

84 Mäkilä P, Hirvensalo M, Parkatti T: Changes in physical activity involvement and attitude to physical activity in a 16-year follow-up study among the elderly. J Aging Res 2010; 2010:174290.

85 Menec VH, Chipperfield JG: Remaining active in later life: the role of locus of control in seniors' leisure activity participation, health, and life satisfaction. J Aging Health 1997;9: 105-125.

86 Merom D, Cosgrove C, Kamalesh V, Bauman A: How diverse was the leisure time physical activity of older Australians over the past decade? J Sci Med Sport 2012;15:213-219.

87 Merom D, Bauman A, Ford I: The public health usefulness of the Exercise Recreation and Sport Survey (ERASS) surveillance system. J Sci Med Sport 2004;7:32-37.
88 Mier N, Smith ML, Carrillo-Zuniga G, Wang X, Garza N, Ory MG: Personal and cultural influences on diabetes self-care behaviors among older Hispanics born in the U.S. and Mexico. J Immigr Minor Health 2012;14:1052-1062.

89 Moschny A, Platen P, Klaassen-Mielke R, Trampisch U, Hinrichs T: Physical activity patterns in older men and women in Germany: a cross-sectional study. BMC Public Health 2011;11:559.

90 Trampisch U, Platen P, Burghaus I, Moschny A, Wilm S, Thiem U, Hinrichs T: Reliability of the PRISCUS-PAQ. Questionnaire to assess physical activity of persons aged 70 years and older (in German). Z Gerontol Geriatr 2010;43:399-406.

91 Murphy MH, Donnelly P, Breslin G, Shibli S, Nevill AM: Does doing housework keep you healthy? The contribution of domestic physical activity to meeting current recommendations for health. BMC Public Health 2013;13:966.

92 Mynarski W, Rozpara M, Nawrocka A, Borek Z, Powerska A, Garbaciak W: Physical activity of middle-age adults aged 50-65 years in view of health recommendations. Eur Rev Aging Phys Act 2014;11:141-147.

93 Palacios-Ceña D, Alonso-Blanco C, Jiménez-Garcia R, Hernández-Barrera V, Carrasco-Garrido P, Pileño-Martinez E, Fernández-de-las-Peñas C: Time trends in leisure time physical activity and physical fitness in elderly people: 20 year follow-up of the Spanish population national health survey (1987-2006). BMC Public Health 2011;11: 799.

94 Persson A, While A: Physical activity among older people and related factors. Health Educ J 2011;71:144-153.

95 Reigal R, Videra A: Frequency of physical activity and self-efficacy in the elderly. Rev Int Med Cienc Act Fis Deporte 2013;13:107120.

96 Resnick B: Functional performance and exercise of older adults in long-term care settings. J Gerontol Nurs 2000;26:7-16.

97 Resnick B: Testing a model of overall activity in older adults. J Aging Phys Act 2001;9: 142-160.

98 DiPietro L, Caspersen C, Ostfeld A, Nadel E: A survey for assessing physical activity among older adults. Med Sci Sports Exerc 1993;25:628-642.

99 Resnick B, Orwig D, Hawkes W, Shardell M, Golden J, Werner M, Magaziner J: The relationship between psychosocial state and exercise behavior of older women 2 months after hip fracture. Rehabil Nurs 2007;32: 139-149.

100 Rowinski R, Dabrowski A, Kostka T: Gardening as the dominant leisure time physical activity (LTPA) of older adults from a postcommunist country. The results of the population-based PolSenior Project from Poland. Arch Gerontol Geriatr 2015;60:486491.
101 Schüz B, Wurm S, Warner LM, Wolff JK, Schwarzer R: Health motives and health behaviour self-regulation in older adults. J Behav Med 2014;37:491-500.

102 Shemesh AA, Rasooly I, Horowitz P, Lemberger J, Ben-Moshe Y, Kachal J, Rosenberg E: Health behaviors and their determinants in multiethnic, active Israeli seniors. Arch Gerontol Geriatr 2008;47:63-77.

103 Simsek H, Doganay S, Budak R, Ucku R: Relationship of socioeconomic status with health behaviors and self-perceived health in the elderly: a community-based study, Turkey. Geriatr Gerontol Int 2014;14:960-968.

104 Siqueira FV, Augusto LA, Silveira DS, Piccini RX, Tomasi E, Hallal PC: Leisure-time physical activity among adult and elderly individuals in Brazil: a countrywide analysis. J Phys Act Health 2011;8:891-897.

105 Sjögren K, Stjernberg L: A gender perspective on factors that influence outdoor recreational physical activity among the elderly. BMC Geriatrics 2010;10:34.

106 Snodgrass JJ, Liebert MA, Cepon-Robins TJ, Barrett TM, Mathur A, Chatterji S, Kowal P: Accelerometer-measured physical activity among older adults in urban India: results of a study on global ageing and adult health substudy. Am J Hum Biol 2016;28:412-420.

107 Stephan Y, Boiche J, Trouilloud D, Deroche T, Sarrazin P: The relation between risk perceptions and physical activity among older adults: a prospective study. Psychol Health 2011;26:887-897.

108 Godin G, Shephard R: A simple method to assess exercise behavior in the community. Can J Appl Sport Sci 1985;10:141-146.

109 Vuillemin A, Oppert JM, Guillemin L, Essermeant AM, Fontvieille P, Galan A, Kriska LM, Hercberg S: Self-administered questionnaire compared with interview to assess past-year physical activity. Med Sci Sports Exerc 2000;32:1119-1124.

110 Stuart CL, Marret J, Kelley GA, Nelson R: Predictors of physical activity in older adults in an independent living retirement community. Am J Geriatr Cardiol 2002;11:160$162,191$.

111 Walsh JM, Pressman AR, Cauley JA, Browner WS: Predictors of physical activity in community-dwelling elderly white women. J Gen Intern Med 2001;16:721-727.

112 Wister AV: The effects of socioeconomic status on exercise and smoking: age-related differences. J Aging Health 1996;8:467-488.

113 Statistics Canada: Health Promotion Survey (HPS). 2007. http://www23.statcan.gc.ca/ $\mathrm{imdb} / \mathrm{p} 2 \mathrm{SV} \cdot \mathrm{pl}$ ? Function $=$ getSurvey\&SD DS $=3828$.

114 Wolinsky FD, Stump TE, Clark DO: Antecedents and consequences of physical activity and exercise among older adults. Gerontologist 1995;35:451-462.

115 Centers for Disease Control and Prevention: National Health Interview Survey. 2016. https://www.cdc.gov/nchs/nhis/. 
116 Yasunaga A, Park H, Watanabe E, Togo F, Park S, Shephard RJ, Aoyagi Y: Development and evaluation of the physical activity questionnaire for elderly Japanese: the $\mathrm{Na}-$ kanojo study. J Aging Phys Act 2007;15:398411.

117 Yusuf HR, Croft JB, Giles WH, Anda RF, Casper ML, Caspersen CJ, Jones DA: Leisure-time physical activity among older adults. United States, 1990. Arch Intern Med 1996;156:1321-1326.

118 Piani A, Schoenborn CA: Health promotion and disease prevention: United States, 1990. Vital Health Stat 10 1993;185:1-88.
119 Zhao G, Ford ES, Li C, Balluz LS: Physical activity in US older adults with diabetes mellitus: prevalence and correlates of meeting physical activity recommendations. J Am Geriatr Soc 2011;59:132-137.

120 Centers for Disease Control and Prevention: Behavioral Risk Factor Surveillance System (BRFSS). 2016. https://www.cdc.gov/brfss/.

121 Stroup DF, Berlin JA, Morton SC, Olkin I, Williamson GD, Rennie D, Moher D, Becker BJ, Sipe TA, Thacker SB: Meta-analysis of observational studies in epidemiology: a proposal for reporting. Meta-analysis Of Observational Studies in Epidemiology (MOOSE) group. JAMA 2000;283:20082012.
122 Von Elm E, Altman DG, Egger M, Pocock SJ, Gøtzsche PC, Vandenbroucke JP; STROBE Initiative: The Strengthening the Reporting of Observational Studies in Epidemiology (STROBE) statement: guidelines for reporting observational studies. Lancet 2007;370: 1453-1457.

123 Allen MS, Walter EE, McDermott MS: Personality and sedentary behavior: a systematic review and meta-analysis. Health Psychol 2017;36:255-263.

124 Brewer NT, DeFrank JT, Gilkey MB: Anticipated regret and health behavior: a metaanalysis. Health Psychol 2016;35:12641275. 